\title{
GAIA: Composition, formation and evolution of the Galaxy
}

\author{
M. A. C. Perryman ${ }^{1}$, K. S. de Boer ${ }^{2}$, G. Gilmore ${ }^{3}$, E. Høg ${ }^{4}$, M. G. Lattanzi ${ }^{5}$, L. Lindegren ${ }^{6}$, X. Luri ${ }^{7}$, \\ F. Mignard ${ }^{8}$, O. Pace ${ }^{9}$, and P. T. de Zeeuw ${ }^{10}$ \\ 1 Astrophysics Division, Space Science Department of ESA, ESTEC, Postbus 299, 2200 AG Noordwijk, \\ The Netherlands \\ 2 Sternwarte Univ. Bonn, Auf dem Hugel 71, 53121 Bonn, Germany \\ 3 University of Cambridge, Institute of Astronomy, Madingley Road, Cambridge CB3 0HA, UK \\ ${ }^{4}$ Copenhagen University Observatory, Juliane Maries Vej 30, 2100 OE Copenhagen, Denmark \\ 5 Osservatorio Astronomico di Torino, Strada Osservatorio 20, 10025 Pino Torinese (TO), Italy \\ ${ }^{6}$ Lund Observatory, Box 43, 22100 Lund, Sweden \\ 7 Universitat de Barcelona, Departament d'Astronomia i Meteorologia, Avda Diagonal 647, 08028 Barcelona, \\ Spain \\ 8 Observatoire de la Côte d'Azur, CERGA, Avenue Copernic, 06130 Grasse, France \\ 9 Future Projects Division of ESA, ESTEC, Postbus 299, 2200 AG Noordwijk, The Netherlands \\ 10 Sterrewacht, Jan Hendrik Oort Building, Postbus 9513, 2300 RA Leiden, The Netherlands
}

Received 1 November 2000 / Accepted 5 January 2001

\begin{abstract}
The GAIA astrometric mission has recently been approved as one of the next two "cornerstones" of ESA's science programme, with a launch date target of not later than mid-2012. GAIA will provide positional and radial velocity measurements with the accuracies needed to produce a stereoscopic and kinematic census of about one billion stars throughout our Galaxy (and into the Local Group), amounting to about 1 percent of the Galactic stellar population. GAIA's main scientific goal is to clarify the origin and history of our Galaxy, from a quantitative census of the stellar populations. It will advance questions such as when the stars in our Galaxy formed, when and how it was assembled, and its distribution of dark matter. The survey aims for completeness to $V=20 \mathrm{mag}$, with accuracies of about $10 \mu \mathrm{as}$ at $15 \mathrm{mag}$. Combined with astrophysical information for each star, provided by on-board multi-colour photometry and (limited) spectroscopy, these data will have the precision necessary to quantify the early formation, and subsequent dynamical, chemical and star formation evolution of our Galaxy. Additional products include detection and orbital classification of tens of thousands of extra-Solar planetary systems, and a comprehensive survey of some $10^{5}-10^{6}$ minor bodies in our Solar System, through galaxies in the nearby Universe, to some 500000 distant quasars. It will provide a number of stringent new tests of general relativity and cosmology. The complete satellite system was evaluated as part of a detailed technology study, including a detailed payload design, corresponding accuracy assesments, and results from a prototype data reduction development.
\end{abstract}

Key words. instrumentation: miscellaneous - space vehicles: instruments - astrometry - galaxy: general techniques: photometric - techniques: radial velocities

\section{Introduction}

Understanding the details of the Galaxy in which we live is one of the great intellectual challenges embraced by modern science. Our Galaxy contains a complex mix of stars, planets, interstellar gas and dust, radiation, and the ubiquitous dark matter. These components are widely distributed in age (reflecting their birth rate), in space (reflecting their birth places and subsequent motions), on orbits (determined by the gravitational force generated by their own mass), and with complex distributions of

Send offprint requests to: M. A. C. Perryman, e-mail: mperryma@astro.estec.esa.nl chemical element abundances (determined by the past history of star formation and gas accretion).

Astrophysics has now developed the tools to measure these distributions in space, kinematics, and chemical abundance, and to interpret the distribution functions to map, and to understand, the formation, structure, evolution, and future of our entire Galaxy. This potential understanding is also of profound significance for quantitative studies of the high-redshift Universe: a well-studied nearby template galaxy would underpin the analysis of unresolved galaxies with other facilities, and at other wavelengths.

Understanding the structure and evolution of the Galaxy requires three complementary observational approaches: (i) a census of the contents of a large, 
representative, part of the Galaxy; (ii) quantification of the present spatial structure, from distances; (iii) knowledge of the three-dimensional space motions, to determine the gravitational field and the stellar orbits. Astrometric measurements uniquely provide model-independent distances and transverse kinematics, and form the basis of the cosmic distance scale. Complementary radial velocity and photometric information are required to complete the kinematic and astrophysical information about the individual objects observed.

Photometry, with appropriate astrometric and astrophysical calibration, gives a knowledge of extinction, and hence, combined with astrometry, provides intrinsic luminosities, spatial distribution functions, and stellar chemical abundance and age information. Radial velocities complete the kinematic triad, allowing determination of dynamical motions, gravitational forces, and the distribution of invisible mass. The GAIA mission will provide all this information.

Even before the end of the Hipparcos mission, a proposal for an ambitious follow-on space astrometry experiment was submitted to ESA (Roemer: Høg 1993; Lindegren et al. 1993a; Høg \& Lindegren 1994). The idea of using CCDs as a modulation detector behind a grid (Høg \& Lindegren 1993), similar to Hipparcos, was replaced by the more powerful option adopted for Roemer (Høg 1993) where CCDs measure the direct stellar images in time-delayed integration (TDI) mode in the scanning satellite. A more ambitious interferometric mission, GAIA, was proposed and subsequently recommended as a cornerstone mission of the ESA science programme by the Horizon 2000+ Survey Committee in 1994 (Battrick 1994). The GAIA proposal demonstrated that accuracies of $10 \mu \mathrm{as}$ at $15 \mathrm{mag}$ were achievable using a small interferometer (Lindegren et al. 1993b; Lindegren \& Perryman 1996).

The European scientific community and ESA have now completed a detailed study of the science case and instrument design, identifying a number of further improvements, including reverting to full-aperture telescopes ( $\mathrm{H} ø \mathrm{~g}$ 1995a; Høg 1995b). The results demonstrate that unique and fundamental advances in astrophysics are technically achievable on the proposed time-scales, and within a budget profile consistent with the current ESA cornerstone mission financial envelope.

GAIA will be a continuously scanning spacecraft, accurately measuring one-dimensional coordinates along great circles in two simultaneous fields of view, separated by a well-known angle. The payload utilises a large but feasible CCD focal plane assembly, passive thermal control, natural short-term instrument stability due to the Sun shield and the selected orbit, and a robust payload design. The telescopes are of moderate size, with no specific manufacturing complexity. The system fits within a dual-launch Ariane 5 configuration, without deployment of any payload elements. The study identifies a "Lissajous" orbit at L2 as the preferred operational orbit, from where about 1 Mbit of data per second is returned to the single ground station throughout the 5-year mission. A comprehensive accuracy assessment has validated the proposed payload and the subsequent data reduction.

This paper provides a summary of the key features of the improved GAIA design, and the resulting scientific case, evaluated during the recent study phase (ESA 2000; see also http: //astro.estec.esa.nl/GAIA). A comparison between the scientific goals of GAIA, and other postHipparcos space astrometry missions, is given in Sect. 8 .

\section{Scientific goals}

\subsection{Structure and dynamics of the galaxy}

The primary objective of the GAIA mission is to observe the physical characteristics, kinematics and distribution of stars over a large fraction of the volume of our Galaxy, with the goal of achieving a full understanding of its dynamics and structure, and consequently its formation and history (see, e.g., Gilmore et al. 1989; Majewski 1993; Ibata et al. 1997; Wyse et al. 1997; de Zeeuw 1999; as well as extensive details of the scientific case given in ESA 2000). An overview of the main Galaxy components and sub-populations is given in Table 1, together with requirements on astrometric accuracy and limiting magnitude.

\subsection{The star formation history of our Galaxy}

A central element the GAIA mission is the determination of the star formation histories, as described by the temporal evolution of the star formation rate, and the cumulative numbers of stars formed, of the bulge, inner disk, Solar neighbourhood, outer disk and halo of our Galaxy (e.g. Hernandez et al. 2000). Given such information, together with the kinematic information from GAIA, and complementary chemical abundance information, again primarily from GAIA, the full evolutionary history of the Galaxy is determinable (e.g. Freeman 1993; Gilmore 1999).

Determination of the relative rates of formation of the stellar populations in a large spiral, typical of those galaxies which dominate the luminosity in the Universe, will provide for the first time quantitative tests of galaxy formation models. Do large galaxies form from accumulation of many smaller systems which have already initiated star formation? Does star formation begin in a gravitational potential well in which much of the gas is already accumulated? Does the bulge pre-date, postdate, or is it contemporaneous with, the halo and inner disk? Is the thick disk a mix of the early disk and a later major merger? Is there a radial age gradient in the older stars? Is the history of star formation relatively smooth, or highly episodic? Answers to such questions will also provide a template for analysis of data on unresolved stellar systems, where similar data cannot be obtained. 
Table 1. Some Galactic kinematic tracers, with corresponding limiting magnitudes and required astrometric accuracy. For various tracers (Col. 1), Cols. 2-6 indicate relevant values of parameters leading to the typical range of $V$ magnitudes over which the populations must be sampled (Cols. 7-8). These results demonstrate that the faint magnitude limit of GAIA is essential for probing the different Galaxy populations, while the astrometry accuracies in Cols. 9-12 demonstrate that GAIA will meet the scientific goals (based on Gilmore \& Høg 1995)

\begin{tabular}{|c|c|c|c|c|c|c|c|c|c|c|c|}
\hline (1) & $(2)$ & $(3)$ & (4) & $(5)$ & $(6)$ & $(7)$ & $(8)$ & $(9)$ & $(10)$ & $(11)$ & $(12)$ \\
\hline Tracer & $\begin{array}{l}M_{V} \\
\text { mag }\end{array}$ & $\begin{array}{c}\ell \\
\operatorname{deg}\end{array}$ & $\begin{array}{c}b \\
\operatorname{deg}\end{array}$ & $\begin{array}{c}d \\
\mathrm{kpc}\end{array}$ & $\begin{array}{r}A_{V} \\
\text { mag }\end{array}$ & $\begin{array}{c}V_{1} \\
\text { mag }\end{array}$ & $\begin{array}{c}V_{2} \\
\text { mag }\end{array}$ & $\begin{array}{c}\epsilon_{\mathrm{T}} \\
\mathrm{km} \mathrm{s}^{-1}\end{array}$ & $\begin{array}{c}\sigma_{\mu_{1}} \\
\mu \mathrm{as} / \mathrm{yr}\end{array}$ & $\begin{array}{c}\sigma_{\mu_{1}}^{\prime} \\
-\end{array}$ & $\begin{array}{c}\sigma_{\pi_{1}}^{\prime} \\
-\end{array}$ \\
\hline \multicolumn{12}{|l|}{ Bulge: } \\
\hline $\mathrm{gM}$ & -1 & 0 & $<20$ & 8 & $2-10$ & 15 & 20 & 100 & 10 & 0.01 & 0.10 \\
\hline $\mathrm{HB}$ & +0.5 & 0 & $<20$ & 8 & $2-10$ & 17 & 20 & 100 & 20 & 0.01 & 0.20 \\
\hline MS turnoff & +4.5 & 1 & -4 & 8 & $0-2$ & 19 & 21 & 100 & 60 & 0.02 & 0.6 \\
\hline \multicolumn{12}{|l|}{ Spiral arms: } \\
\hline Cepheids & -4 & all & $<10$ & 10 & $3-7$ & 14 & 18 & 7 & 5 & 0.03 & 0.06 \\
\hline B-M Supergiants & -5 & all & $<10$ & 10 & $3-7$ & 13 & 17 & 7 & 4 & 0.03 & 0.05 \\
\hline Perseus arm (B) & -2 & 140 & $<10$ & 2 & $2-6$ & 12 & 16 & 10 & 3 & 0.01 & 0.01 \\
\hline \multicolumn{12}{|l|}{ Thin disk: } \\
\hline gK & -1 & 0 & $<15$ & 8 & $1-5$ & 14 & 18 & 40 & 6 & 0.01 & 0.06 \\
\hline gK & -1 & 180 & $<15$ & 10 & $1-5$ & 15 & 19 & 10 & 8 & 0.04 & 0.10 \\
\hline Disk warp (gM) & -1 & all & $<20$ & 10 & $1-5$ & 15 & 19 & 10 & 8 & 0.04 & 0.10 \\
\hline Disk asymmetry (gM) & -1 & all & $<20$ & 20 & $1-5$ & 16 & 20 & 10 & 15 & 0.14 & 0.4 \\
\hline \multicolumn{12}{|l|}{ Thick disk: } \\
\hline Miras, gK & -1 & 0 & $<30$ & 8 & 2 & 15 & 19 & 50 & 10 & 0.01 & 0.10 \\
\hline HB & +0.5 & 0 & $<30$ & 8 & 2 & 15 & 19 & 50 & 20 & 0.02 & 0.20 \\
\hline Miras, gK & -1 & 180 & $<30$ & 20 & 2 & 15 & 21 & 30 & 25 & 0.08 & 0.65 \\
\hline $\mathrm{HB}$ & +0.5 & 180 & $<30$ & 20 & 2 & 15 & 19 & 30 & 60 & 0.20 & 1.5 \\
\hline \multicolumn{12}{|l|}{ Halo: } \\
\hline$g G$ & -1 & all & $<20$ & 8 & $2-3$ & 13 & 21 & 100 & 10 & 0.01 & 0.10 \\
\hline $\mathrm{HB}$ & +0.5 & all & $>20$ & 30 & 0 & 13 & 21 & 100 & 35 & 0.05 & 1.4 \\
\hline \multicolumn{12}{|l|}{ Gravity, $\mathcal{K}_{\mathcal{Z}}$ : } \\
\hline $\mathrm{dK}$ & $+7-8$ & all & all & 2 & 0 & 12 & 20 & 20 & 60 & 0.01 & 0.16 \\
\hline $\mathrm{dF} 8-\mathrm{dG} 2$ & $+5-6$ & all & all & 2 & 0 & 12 & 20 & 20 & 20 & 0.01 & 0.05 \\
\hline Globular clusters (gK) & +1 & all & all & 50 & 0 & 12 & 21 & 100 & 10 & 0.01 & 0.10 \\
\hline internal kinematics (gK) & +1 & all & all & 8 & 0 & 13 & 17 & 15 & 10 & 0.02 & 0.10 \\
\hline Satellite orbits (gM) & -1 & all & all & 100 & 0 & 13 & 20 & 100 & 60 & 0.3 & 8 \\
\hline
\end{tabular}

\subsection{Stellar astrophysics}

GAIA will provide distances of unprecedented accuracy for all types of stars of all stellar populations, even those in the most rapid evolutionary phases which are very sparsely represented in the Solar neighbourhood. All parts of the Hertzsprung-Russell diagram will be comprehensively calibrated, from pre-main sequence stars to white dwarfs and all transient phases; all possible masses, from brown dwarfs to the most massive $\mathrm{O}$ stars; all types of variable stars; all possible types of binary systems down to brown dwarf and planetary systems; all standard distance indicators, etc. This extensive amount of data of extreme accuracy will stimulate a revolution in the exploration of stellar and Galactic formation and evolution, and the determination of the cosmic distance scale (cf. Lebreton 2000).

\subsection{Variability}

The GAIA large-scale photometric survey will have significant intrinsic scientific value for stellar astrophysics, providing valuable samples of variable stars of nearly all types, including detached eclipsing binaries, contact or semi-contact binaries, and pulsating stars (cf. Paczyński 1997). The pulsating stars include key distance calibrators such as Cepheids and RR Lyrae stars and longperiod variables. Existing samples are incomplete already at magnitudes as bright as $V \sim 10$ mag. A complete sample of objects will allow determination of the frequency of variable objects, and will accurately calibrate 
period-luminosity relationships across a wide range of stellar parameters including metallicity. A systematic variability search will also allow identification of stars in short-lived but key stages of stellar evolution, such as the helium core flash and the helium shell thermal pulses and flashes. Prompt processing will identify many targets for follow-up ground-based studies. Estimated numbers are highly uncertain, but suggest some 18 million variable stars in total, including 5 million "classic" periodic variables, 2-3 million eclipsing binaries, 2000-8000 Cepheids, $60000-240000 \delta$ Scuti variables, $70000 \mathrm{RR}$ Lyrae, and 140 000-170000 Miras (Eyer \& Cuypers 2000).

\subsection{Binaries and multiple stars}

A key scientific issue regarding double and multiple star formation is the distribution of mass-ratios $q$. For wide pairs $(>0.5$ arcsec) this is indirectly given through the distribution of magnitude differences. GAIA will provide a photometric determination of the $q$-distribution down to $q \sim 0.1$, covering the expected maximum around $q \sim 0.2$. Furthermore, the large numbers of ("5-year") astrometric orbits, will allow derivation of the important statistics of the very smallest (brown dwarf) masses as well as the detailed distribution of orbital eccentricities (Söderhjelm 1999).

GAIA is extremely sensitive to non-linear proper motions. A large fraction of all astrometric binaries with periods from $0.03-30$ years will be immediately recognized by their poor fit to a standard single-star model. Most will be unresolved, with very unequal mass-ratios and/or magnitudes, but in many cases a photocentre orbit can be determined. For this period range, the absolute and relative binary frequency can be established, with the important possibility of exploring variations with age and place of formation in the Galaxy. Some 10 million binaries closer than $250 \mathrm{pc}$ should be detected, with very much larger numbers still detectable out to $1 \mathrm{kpc}$ and beyond.

\subsection{Brown dwarfs and planetary systems}

Sub-stellar companions can be divided in two classes: brown dwarfs and planets. There exist three major genesis indicators that can help classify sub-stellar objects as either brown dwarfs or planets: mass, shape and alignment of the orbit, and composition and thermal structure of the atmosphere. Mass alone is not decisive. The ability to simultaneously and systematically determine planetary frequency and distribution of orbital parameters for the stellar mix in the Solar neighbourhood is a fundamental contribution that GAIA will uniquely provide. Any changes in planetary frequency with age or metallicity will come from observations of stars of all ages.

An isolated brown dwarf is typically visible only at ages $<1$ Gyr because of their rapidly fading luminosity with time. However, in a binary system, the mass is conserved, and the gravitational effects on a main-sequence secondary remain observable over much longer intervals. GAIA will have the power to investigate the mass-distribution of brown-dwarf binaries with 1-30 year periods, of all ages, through analysis of the astrometric orbits.

There are a number of techniques which in principle allow the detection of extra-Solar planetary systems: these include pulsar timing, radial velocity measurements, astrometric techniques, transit measurements, microlensing, and direct methods based on high-angular resolution interferometric imaging. A better understanding of the conditions under which planetary systems form and of their general properties requires sensitivity to low mass planets (down to $\sim 10 M_{\oplus}$ ), characterization of known systems (mass, and orbital elements), and complete samples of planets, with useful upper limits on Jupiter-mass planets out to several AU from the central star (Marcy \& Butler 1998; Perryman 2000).

Astrometric measurements good to 2-10 $\mu$ as will contribute substantially to these goals, and will complement the ongoing radial velocity measurement programmes. Although SIM will be able to study in detail targets detected by other methods, including microlensing, GAIA's strength will be its discovery potential, following from the astrometric monitoring of all of the several hundred thousand bright stars out to distances of $\sim 200$ pc (Lattanzi et al. 2000).

\subsection{Solar system}

Solar System objects present a challenge to GAIA because of their significant proper motions, but they promise a rich scientific reward. The minor bodies provide a record of the conditions in the proto-Solar nebula, and their properties therefore shed light on the formation of planetary systems.

The relatively small bodies located in the main asteroid belt between Mars and Jupiter should have experienced limited thermal evolution since the early epochs of planetary accretion. Due to the radial extent of the main belt, minor planets provide important information about the gradient of mineralogical composition of the early planetesimals as a function of heliocentric distance. It is therefore important for any study of the origin and evolution of the Solar system to investigate the main physical properties of asteroids including masses, densities, sizes, shapes, and taxonomic classes, all as a function of location in the main belt and in the Trojan clouds.

The possibility of determining asteroid masses relies on the capability of measuring the tiny gravitational perturbations that asteroids experience in case of a mutual close approach. At present only about 10 asteroid masses are known, mostly with quite poor accuracy. Asteroidasteroid encounters have been modelled, and show that GAIA will allow more than 100 asteroid masses to be determined accurately.

Albedo is a useful complement to spectrophotometric data for the definition of different taxonomic classes. 
The GAIA photometry will be much more reliable than most data presently available. The colour indices will provide a taxonomic classification for the whole sample of observed asteroids.

For direct orbit determinations of known asteroids, preliminary simulations have been performed in which the covariance matrices of the orbital elements of more than 6000 asteroids were computed using both the whole set of astrometric observations collected from ground-based telescopes since 1895 through 1995, as well as a set of simulated observations carried out by GAIA, computed by considering a 5 year lifetime of the mission, and present instrument performances. Another set of simulated groundbased observations covering the period 1996-2015 were also performed. For the known asteroids the predicted ephemeris errors based on the GAIA observations alone 100 years after the end of the mission are more than a factor 30 better than the predicted ephemeris errors corresponding to the whole set of past and future groundbased observations. In other words, after the collection of the GAIA data, all the results of more than one century of ground-based asteroid astrometry will be largely superseded.

In addition to known asteroids, GAIA will discover a very large number, of the order of $10^{5}$ or $10^{6}$ new objects, depending on the uncertainties on the extrapolations of the known population. It should be possible to derive precise orbits for many of the newly discovered objects, since each of them will be observed many times during the mission lifetime. These will include a large number of nearEarth asteroids. The combination of on-board detection, faint limiting magnitude, observations at small Sun-aspect angles, high accuracy in the instantaneous angular velocity $\left(0.25\right.$ mas s$\left.^{-1}\right)$, and confirmation from successive field transits, means that GAIA will provide a detailed census of Atens, Apollos and Amors, extending as close as $0.5 \mathrm{AU}$ to the Sun, and down to diameters of about $260-590 \mathrm{~m}$ at $1 \mathrm{AU}$, depending on albedo and observational geometry.

\subsection{Galaxies, quasars, and the reference frame}

GAIA will not only provide a representative census of the stars throughout the Galaxy, but it will also make unique contributions to extragalactic astronomy (Table 2). These include the structure, dynamics and stellar populations in the Local Group, especially the Magellanic Clouds, M 31 and M33, the space motions of Local Group galaxies, a multi-colour survey of galaxies (Vaccari 2000), and studies of supernovae (Høg et al. 1999b), galactic nuclei, and quasars.

\subsection{The radio/optical reference frame}

The International Celestial Reference System (ICRS) is realized by the International Celestial Reference Frame (ICRF) consisting of 212 extragalactic radio-sources with an rms uncertainty in position between 100 and $500 \mu$ as.
The extension of the ICRF to visible light is represented by the Hipparcos Catalogue. This has rms uncertainties estimated to be 0.25 mas $\mathrm{yr}^{-1}$ in each component of the spin vector of the frame, and 0.6 mas in the components of the orientation vector at the catalogue epoch, J1991.25. The GAIA catalogue will permit a definition of the ICRS more accurate by one or two orders of magnitude than the present realizations (e.g. Feissel \& Mignard 1998; Johnston \& de Vegt 1999).

The spin vector can be determined very accurately by means of the many thousand faint quasars picked up by the astrometric and photometric survey. Simulations using realistic quasar counts, conservative estimates of intrinsic source photocentric instability, and realistic intervening gravitational lensing effects, show that an accuracy of better than $0.4 \mu \mathrm{as} \mathrm{yr}^{-1}$ will be reached in all three components of the spin vector.

For the determination of the frame orientation, the only possible procedure is to compare the positions of the radio sources in ICRF (and its extensions) with the positions of their optical counterparts observed by GAIA. The number of such objects is currently less than 300 and the error budget is dominated by the uncertainties of the radio positions. Assuming current accuracies for the radio positions, simulations show that the GAIA frame orientation will be obtained with an uncertainty of $\sim 60 \mu$ as in each component of the orientation vector. The actual result by the time of GAIA may be significantly better, as the number and quality of radio positions for suitable objects are likely to increase with time.

The Sun's absolute velocity with respect to a cosmological reference frame causes the dipole anisotropy of the cosmic microwave background. The Sun's absolute acceleration can be measured astrometrically: it will result in the apparent proper motion of quasars. The acceleration of the Solar System towards the Galactic centre causes the aberration effect to change slowly. This leads to a slow change of the apparent positions of distant celestial objects, i.e., to an apparent proper motion. For a Solar velocity of $220 \mathrm{~km} \mathrm{~s}^{-1}$ and a distance of $8.5 \mathrm{kpc}$ to the Galactic centre, the orbital period of the Sun is $\sim 250 \mathrm{Myr}$, and the Galactocentric acceleration has the value $0.2 \mathrm{~nm} \mathrm{~s}^{-2}$, or $6 \mathrm{~mm} \mathrm{~s}^{-1} \mathrm{yr}^{-1}$. A change in velocity by $6 \mathrm{~mm} \mathrm{~s}^{-1}$ causes a change in aberration of the order of $4 \mu \mathrm{as}$. The apparent proper motion of a celestial object caused by this effect always points towards the direction of the Galactic centre. Thus, all quasars will exhibit a streaming motion towards the Galactic centre of this amplitude.

\subsection{Fundamental physics}

The reduction of the Hipparcos data necessitated the inclusion of stellar aberration up to terms in $(v / c)^{2}$, and the general relativistic treatment of light bending due to the gravitational field of the Sun (and Earth). The GAIA data reduction requires a more accurate and comprehensive inclusion of relativistic effects, at the same time providing 
Table 2. Local Group galaxies potentially accessible to GAIA. $E(B-V)$ indicates the foreground reddening, and $(m-M)_{0}$ is the true distance modulus. $V_{\mathrm{lim}}$ is the brightest star in the galaxy. $\mu_{v_{\mathrm{t}}-v_{\mathrm{r}}}$ is the estimated proper motion, assuming the transverse velocity equals the observed radial velocity. ${ }^{*}$ denotes observed values

\begin{tabular}{|c|c|c|c|c|c|c|c|c|c|}
\hline Galaxy & $\begin{array}{r}l \\
\left({ }^{\circ}\right)\end{array}$ & $\begin{array}{r}b \\
\left({ }^{\circ}\right) \\
\end{array}$ & $\begin{array}{l}E(B-V) \\
(\mathrm{mag})\end{array}$ & $\begin{array}{l}(m-M)_{0} \\
(\mathrm{mag})\end{array}$ & $\begin{array}{c}\text { Distance } \\
(\mathrm{kpc})\end{array}$ & $\begin{array}{c}V_{\lim } \\
(\mathrm{mag})\end{array}$ & $\begin{array}{l}N(\text { stars }) \\
(V<20)\end{array}$ & $\begin{array}{r}V_{r} \\
\text { (helio) }\end{array}$ & $\begin{array}{r}\mu_{v_{\mathrm{t}}-v_{\mathrm{r}}} \\
(\mu \mathrm{as} / \mathrm{yr})\end{array}$ \\
\hline WLM & 75.9 & -73.6 & $0.02 \pm 0.01$ & $24.83 \pm 0.08$ & $925 \pm 40$ & 16.5 & $\sim 500$ & -116 & 26 \\
\hline NGC 55 & 332.7 & -75.7 & $0.03 \pm 0.02$ & $25.85 \pm 0.20$ & $1480 \pm 150$ & 15.0 & 10 's & 129 & 18 \\
\hline IC 10 & 119.0 & -3.3 & $0.87 \pm 0.12$ & $24.58 \pm 0.12$ & $825 \pm 50$ & 15.0 & 10 's & -344 & 83 \\
\hline NGC 147 & 119.8 & -14.3 & $0.18 \pm 0.03$ & $24.30 \pm 0.12$ & $725 \pm 45$ & 18.5 & 10 's & -193 & 56 \\
\hline And III & 119.3 & -26.2 & $0.05 \pm 0.02$ & $24.40 \pm 0.10$ & $760 \pm 40$ & 20 & & & 60 \\
\hline NGC 185 & 120.8 & -14.5 & $0.19 \pm 0.02$ & $23.96 \pm 0.08$ & $620 \pm 25$ & 20 & & -202 & 69 \\
\hline NGC 205 & 120.7 & -21.7 & $0.04 \pm 0.02$ & $24.56 \pm 0.08$ & $815 \pm 35$ & 20 & & -241 & 62 \\
\hline M 32 & 121.2 & -22.0 & $0.08 \pm 0.03$ & $24.53 \pm 0.08$ & $805 \pm 35$ & 16 & $\sim 10^{4}$ & -205 & 54 \\
\hline M 31 & 121.2 & -21.6 & 0.08 & 24.43 & 770 & 15 & $\gg 10^{4}$ & -297 & 81 \\
\hline And I & 121.7 & -24.9 & $0.04 \pm 0.02$ & $24.53 \pm 0.10$ & $805 \pm 40$ & 21.7 & & & \\
\hline $\mathrm{SMC}$ & 302.8 & -44.3 & 0.08 & 18.82 & 58 & 12 & $>10^{6}$ & 158 & $900^{*}$ \\
\hline Sculptor & 287.5 & -83.2 & $0.02 \pm 0.02$ & $19.54 \pm 0.08$ & $79 \pm 4$ & 16.0 & 100's & 110 & $360^{*}$ \\
\hline LGS 3 & 126.8 & -40.9 & $0.08 \pm 0.03$ & $24.54 \pm 0.15$ & $810 \pm 60$ & & & -277 & 72 \\
\hline IC 1613 & 129.8 & -60.6 & $0.03 \pm 0.02$ & $24.22 \pm 0.10$ & $700 \pm 35$ & 17.1 & 100 's & -234 & 71 \\
\hline And II & 128.9 & -29.2 & $0.08 \pm 0.02$ & $23.6 \pm 0.4$ & $525 \pm 110$ & 20 & & & \\
\hline M 33 & 133.6 & -31.3 & 0.08 & 24.62 & 840 & 15 & $>10^{4}$ & -181 & 46 \\
\hline Phoenix & 272.2 & -68.9 & $0.02 \pm 0.01$ & $23.24 \pm 0.12$ & $445 \pm 30$ & 17.9 & $\sim 10^{2}$ & 56 & 27 \\
\hline Fornax & 237.1 & -65.7 & $0.03 \pm 0.01$ & $20.70 \pm 0.12$ & $138 \pm 8$ & 14 & 100 's & 53 & 81 \\
\hline EGB $0427+63$ & 144.7 & -10.5 & $0.30 \pm 0.15$ & $25.6 \pm 0.7$ & $1300 \pm 700$ & & & -99 & 16 \\
\hline LMC & 280.5 & -32.9 & 0.06 & 18.45 & 49 & 12 & $>10^{7}$ & 278 & $1150^{*}$ \\
\hline Carina & 260.1 & -22.2 & $0.04 \pm 0.02$ & $20.03 \pm 0.09$ & $101 \pm 5$ & 18 & $\sim 10^{3}$ & 229 & 478 \\
\hline Leo A & 196.9 & +52.4 & $0.01 \pm 0.01$ & $24.2 \pm 0.3$ & $690 \pm 100$ & & & 20 & 6 \\
\hline Sextans B & 233.2 & +43.8 & $0.01 \pm 0.02$ & $25.64 \pm 0.15$ & $1345 \pm 100$ & 19.0 & 10 's & 301 & 47 \\
\hline NGC 3109 & 262.1 & +23.1 & $0.04 \pm 0.02$ & $25.48 \pm 0.25$ & $1250 \pm 165$ & & & 403 & 68 \\
\hline Antlia & 263.1 & +22.3 & $0.05 \pm 0.03$ & $25.46 \pm 0.10$ & $1235 \pm 65$ & & & 361 & 62 \\
\hline Leo I & 226.0 & +49.1 & $0.01 \pm 0.01$ & $21.99 \pm 0.20$ & $250 \pm 30$ & 19 & 10 's & 168 & 142 \\
\hline Sextans A & 246.2 & +39.9 & $0.03 \pm 0.02$ & $25.75 \pm 0.15$ & $1440 \pm 110$ & 17.5 & 10 's & 324 & 48 \\
\hline Sextans & 243.5 & +42.3 & $0.03 \pm 0.01$ & $19.67 \pm 0.08$ & $86 \pm 4$ & & & 230 & 564 \\
\hline Leo II & 220.2 & +67.2 & $0.02 \pm 0.01$ & $21.63 \pm 0.09$ & $205 \pm 12$ & 18.6 & 100 's & 90 & 95 \\
\hline GR 8 & 310.7 & +77.0 & $0.02 \pm 0.02$ & $25.9 \pm 0.4$ & $1510 \pm 330$ & 18.7 & 10 's & 214 & 28 \\
\hline Ursa Minor & 105.0 & +44.8 & $0.03 \pm 0.02$ & $19.11 \pm 0.10$ & $66 \pm 3$ & 16.9 & 100 's & -209 & $1000^{*}$ \\
\hline Draco & 86.4 & +34.7 & $0.03 \pm 0.01$ & $19.58 \pm 0.15$ & $82 \pm 6$ & 17 & 100 's & -281 & $1000^{*}$ \\
\hline Sagittarius & 5.6 & -14.1 & $0.15 \pm 0.03$ & $16.90 \pm 0.15$ & $24 \pm 2$ & 14 & $>10^{4}$ & 140 & $2100^{*}$ \\
\hline SagDIG & 21.1 & -16.3 & $0.22 \pm 0.06$ & $25.2 \pm 0.3$ & $1060 \pm 160$ & & & -77 & 16 \\
\hline NGC 6822 & 25.3 & -18.4 & $0.26 \pm 0.04$ & $23.45 \pm 0.15$ & $490 \pm 40$ & & & -57 & 25 \\
\hline DDO 210 & 34.0 & -31.3 & $0.06 \pm 0.02$ & $24.6 \pm 0.5$ & $800 \pm 250$ & 18.9 & 10 's & -137 & 36 \\
\hline IC 5152 & 343.9 & -50.2 & $0.01 \pm 0.02$ & $26.01 \pm 0.25$ & $1590 \pm 200$ & & & 124 & 16 \\
\hline Tucana & 322.9 & -47.4 & $0.00 \pm 0.02$ & $24.73 \pm 0.08$ & $880 \pm 40$ & 18.5 & 10 's & & \\
\hline Pegasus & 94.8 & -43.5 & $0.02 \pm 0.01$ & $24.90 \pm 0.10$ & $955 \pm 50$ & 20 & & -183 & 40 \\
\hline
\end{tabular}

the opportunity to test a number of parameters of general relativity in new observational domains, and with much improved precision.

The dominant relativistic effect in the GAIA measurements is gravitational light bending, quantified by, and allowing accurate determination of, the parameter $\gamma$ of the Parametrized Post-Newtonian (PPN) formulation of gravitational theories. This is of key importance in fundamental physics. The Pound-Rebka experiment verified the relativistic prediction of a gravitational redshift for 
Table 3. Light deflection by masses in the Solar System. The monopole effect dominates, and is summarized in the left columns for grazing incidence and for typical values of the angular separation. Columns $\chi_{\min }$ and $\chi_{\max }$ give results for the minimum and maximum angles accessible to GAIA. $\mathrm{J}_{2}$ is the quadrupole moment. The magnitude of the quadrupole effect is given for grazing incidence, and for an angle of $1^{\circ}$. For GAIA this applies only to Jupiter and Saturn, as it will be located at L2, with minimum Sun/Earth avoidance angle of $35^{\circ}$

\begin{tabular}{|c|c|c|c|c|c|c|c|c|c|}
\hline \multirow[t]{2}{*}{ Object } & \multicolumn{6}{|c|}{ Monopole term } & \multicolumn{3}{|c|}{ Quadrupole term } \\
\hline & & $\begin{array}{r}\text { Grazing } \\
(\mu \mathrm{as})\end{array}$ & $\begin{array}{l}\chi_{\min } \\
(\mu \mathrm{as})\end{array}$ & $\begin{array}{r}\chi=45^{\circ} \\
(\mu \mathrm{as})\end{array}$ & $\begin{array}{r}\chi=90^{\circ} \\
(\mu \mathrm{as})\end{array}$ & $\begin{array}{l}\chi_{\max } \\
(\mu \mathrm{as})\end{array}$ & $J_{2}$ & $\begin{array}{r}\text { Grazing } \\
(\mu \text { as })\end{array}$ & $\begin{array}{l}\chi=1^{\circ} \\
(\mu \mathrm{as})\end{array}$ \\
\hline Sun & 1 & 750000 & 13000 & 10000 & 4100 & 2100 & $\leq 10^{-7}$ & 0.3 & - \\
\hline Earth & & 500 & 3 & 2.5 & 1.1 & 0 & 0.001 & 1 & - \\
\hline Jupiter & & 16000 & 16000 & 2.0 & 0.7 & 0 & 0.015 & 500 & $710^{-5}$ \\
\hline Saturn & & 6000 & 6000 & 0.3 & 0.1 & 0 & 0.016 & 200 & $310^{-6}$ \\
\hline
\end{tabular}

photons, an effect probing the time-time component of the metric tensor. Light deflection depends on both the timespace and space-space components. It has been observed on distance scales of $10^{9}-10^{21} \mathrm{~m}$, and on mass scales from $1-10^{13} M_{\odot}$. GAIA will extend the domain of observations by two orders of magnitude in length, and six orders of magnitude in mass.

Table 3 gives the magnitude of the deflection for the Sun and the major planets, at different values of the angular separation $\chi$, for both monopole and quadrupole terms. While $\chi$ is never smaller than $35^{\circ}$ for the Sun (a constraint from GAIA's orbit), grazing incidence is possible for the planets. With the astrometric accuracy of a few $\mu$ as, the magnitude of the expected effects is considerable for the Sun, and also for observations near planets. The GAIA astrometric residuals can be tested for any discrepancies with the prescriptions of general relativity. Detailed analyses indicate that the GAIA measurements will provide a precision of about $510^{-7}$ for $\gamma$, based on multiple observations of $\sim 10^{7}$ stars with $V<13$ mag at wide angles from the Sun, with individual measurement accuracies better than $10 \mu$ as.

Recent developments in cosmology (e.g. inflationary models) and elementary-particle physics (e.g. string theory and Kaluza-Klein theories), consider scalar-tensor theories as plausible alternatives to general relativity. A large class of such theories contain an attractor mechanism towards general relativity in a cosmological sense; if this is how the Universe is evolving, then today we can expect discrepancies of the order of $|\gamma-1| \sim 10^{-7}-10^{-5}$ depending on the theory. This kind of argument provides a strong motivation for any experiments able to reach these accuracies.

GAIA will observe and discover several hundred thousand minor planets during its five year mission. Most of these will belong to the asteroidal main belt, with small orbital eccentricity and semi-major axes close to 3 AU. The members of the Apollo and Aten groups, which are all Earth-orbit crossers, will include objects with semimajor axes of the order of $1 \mathrm{AU}$ and eccentricities as large as 0.9. The Amor group have perihelia between 1-1.3 AU, and approach the Earth but do not cross its orbit.
Table 4. Perihelion precession due to general relativity and the Solar quadrupole moment for a few representative objects. $a=$ semi-major axis; $e=$ eccentricity $G R=$ perihelion precession in mas/yr due to general relativity; $J_{2}=$ perihelion precession in mas/yr due to the Solar quadrupole moment (assuming $J_{2}=10^{-6}$ )

\begin{tabular}{|c|c|c|c|c|}
\hline Body & $\begin{array}{c}a \\
(\mathrm{AU})\end{array}$ & $e$ & $\begin{array}{c}G R \\
\text { (mas/yr) }\end{array}$ & $\begin{array}{c}J_{2} \\
\text { (mas/yr) }\end{array}$ \\
\hline Mercury & 0.39 & 0.21 & 423 & 1.24 \\
\hline Asteroids & 2.7 & 0.1 & 3.4 & 0.001 \\
\hline 1566 Icarus & 1.08 & 0.83 & 102 & 0.30 \\
\hline 5786 Talos & 1.08 & 0.83 & 102 & 0.30 \\
\hline 3200 Phaeton & 1.27 & 0.89 & 103 & 0.40 \\
\hline
\end{tabular}

Relativistic effects and the Solar quadrupole cause the orbital perihelion of a main belt asteroid to precess at a rate about seven times smaller than for Mercury in rate per revolution, although more than a hundred times in absolute rate.

Three cases of Earth-crossing asteroids are considered in Table 4 giving perihelia precession larger than Mercury, due to a favorable combination of distance and eccentricity. The diameters are of the order of $1 \mathrm{~km}$ for Icarus and Talos and $4 \mathrm{~km}$ for Phaeton. Observed at a geocentric distance of $1 \mathrm{AU}$, these objects have a magnitude between $V=15-17 \mathrm{mag}$ and an angular diameter of 4 mas and 1 mas respectively. Thus the astrometric measurements will be of good quality, virtually unaffected by the finite size of the source. A determination of $\lambda$ with an accuracy of $10^{-4}$ is a reasonable goal, with a value closer to $10^{-5}$ probably attainable from the statistics on several tens of planets. An independent determination of the Solar quadrupole moment $J_{2}$ requires good sampling in $a\left(1-e^{2}\right)$, and one can expect a result better than $10^{-7}$.

Revival of interest in the Brans-Dicke-like theories, with a variable $G$, was partially motivated by the appearance of superstring theories where $G$ is considered to be a dynamical quantity. Using the white dwarf luminosity function an upper bound of $\dot{G} / G \leq-(1 \pm 1) 10^{-11} \mathrm{yr}^{-1}$ has been derived, which is comparable to bounds derived 
from the binary pulsar PSR $1913+16$. Since this is a statistical upper limit, any improvement in our knowledge of the white dwarf luminosity function of the Galactic disk will translate into a more stringent upper bound for $\dot{G} / G$. Since GAIA will detect numerous white dwarfs at low luminosities, present errors can be reduced by a factor of roughly 5. If a reliable age of the Solar neighbourhood independent of the white dwarf luminosity function is determinable, the upper limit could be decreased to $10^{-12}-10^{-13} \mathrm{yr}^{-1}$.

\subsection{Summary}

With a census of the accurate positions, distances, space motions (proper motions and radial velocities), and photometry of all approximately one billion objects complete to $V=20$ mag, GAIA's scientific goals are immense, and can be broadly classified as follows:

The Galaxy: origin and history of our Galaxy; tests of hierarchical structure formation theories; star formation history; chemical evolution; inner bulge/bar dynamics; disk/halo interactions; dynamical evolution; nature of the warp; star cluster disruption; dynamics of spiral structure; distribution of dust; distribution of invisible mass; detection of tidally disrupted debris; Galaxy rotation curve; disk mass profile.

Star formation and evolution: in situ luminosity function; dynamics of star forming regions; luminosity function for pre-main sequence stars; detection and categorization of rapid evolutionary phases; complete and detailed local census down to single brown dwarfs; identification/dating of oldest halo white dwarfs; age census; census of binaries and multiple stars.

Distance scale and reference frame: parallax calibration of all distance scale indicators; absolute luminosities of Cepheids; distance to the Magellanic Clouds; definition of the local, kinematically non-rotating metric.

Local Group and beyond: rotational parallaxes for Local Group galaxies; kinematical separation of stellar populations; galaxy orbits and cosmological history; zero proper motion quasar survey; cosmological acceleration of Solar System; photometry of galaxies; detection of supernovae.

Solar System: deep and uniform detection of minor planets; taxonomy and evolution; inner Trojans; Kuiper Belt Objects; near-Earth asteroids; disruption of Oort Cloud.

Extra-Solar planetary systems: complete census of large planets to 200-500 pc; masses; orbital characteristics of several thousand systems; relative orbital inclinations of multiple systems.

Fundamental physics: $\gamma$ to $\sim 5 \quad 10^{-7} ; \beta$ to $310^{-4}-310^{-5}$; Solar $J_{2}$ to $10^{-7}-10^{-8} ; \dot{G} / G$ to $10^{-12}-10^{-13} \mathrm{yr}^{-1}$; constraints on gravitational wave energy for $10^{-12}<f<410^{-9} \mathrm{~Hz}$; constraints on $\Omega_{\mathrm{M}}$ and $\Omega_{\Lambda}$ from quasar microlensing.
Examples of specific objects: $10^{6}-10^{7}$ resolved galaxies; $10^{5}$ extragalactic supernovae; 500000 quasars; $10^{5}-10^{6}$ (new) Solar System objects; $\gtrsim 50000$ brown dwarfs; 30000 extra-Solar planets; 200000 disk white dwarfs; 200 microlensed events; $10^{7}$ resolved binaries within 250 pc.

\section{Overall design considerations}

Instrument design converges through a consideration of technical feasibility and scientific requirements. The proposed GAIA design has arisen from requirements on astrometric precision $(10 \mu$ as at $15 \mathrm{mag})$, completeness to $V=20 \mathrm{mag}$, the acquisition of radial velocities, the provision of accurate multi-colour photometry for astrophysical diagnostics, and the need for on-board object detection (Mignard 1999; Gilmore et al. 2000).

\subsection{Astrometry}

A space astrometry mission has a unique capability to perform global measurements, such that positions, and changes in positions caused by proper motion and parallax, are determined in a reference system consistently defined over the whole sky, for very large numbers of objects. Hipparcos demonstrated that this can be achieved with milliarcsecond accuracy by means of a continuously scanning satellite which observes two directions simultaneously. With current technology this same principle can be applied with a gain of a factor of more than 100 improvement in accuracy, a factor 1000 improvement in limiting magnitude, and a factor of 10000 in the numbers of stars observed.

Measurements conducted by a continuously scanning satellite are optimally efficient, with each photon acquired during a scan contributing to the precision of the resulting astrometric parameters. The over-riding benefit of global astrometry using a scanning satellite is however not efficiency but reliability: an accurate instrument calibration is performed naturally, while the interconnection of observations over the celestial sphere provides the rigidity and reference system, immediately connected to an extragalactic reference system, and a realistic determination of the standard errors of the astrometric parameters. Two individual viewing directions with a wide separation is the fundamental pre-requisite of the payload, since this leads to the determination of absolute trigonometric parallaxes, and absolute distances, exploiting the method implemented for the first time in the Hipparcos mission.

The ultimate accuracy with which the direction to a point source of light can be determined is set by the dual nature of electromagnetic radiation, namely as waves (causing diffraction) and particles (causing a finite signalto-noise ratio in the detection process). For wavelength $\lambda$ and telescope aperture $D$ the characteristic angular size of the diffraction pattern image is of order $\lambda / D$ radians. If a total of $N$ detected photons are available for localizing the image, then the theoretically achievable angular 
accuracy will be of order $(\lambda / D) \times N^{-1 / 2}$ radians. A realistic size for non-deployable space instruments is of order $2 \mathrm{~m}$. Operating in visible light $(\lambda \sim 0.5 \mu \mathrm{m})$ then gives diffraction features of order $\lambda / D \sim 0.05$ arcsec. To achieve a final astrometric accuracy of $10 \mu$ as it is therefore necessary that the diffraction features are localised to within $1 / 5000$ of their characteristic size. Thus, some 25 million detected photons are needed to overcome the statistical noise, although extreme care will be needed to achieve such precision in practice. The requirement on the number of photons can be satisfied for objects around 15 mag with reasonable assumptions on collecting area and bandwidth. Quantifying the tradeoff between dilute versus filled apertures, allowing for attainable focal lengths, attainable pixel sizes, component alignment and stability, and data rates, has clearly pointed in the direction of a moderately large filled aperture (as apposed to an interferometric design) as the optical system of choice.

The GAIA performance target is $10 \mu$ as at $15 \mathrm{mag}$. Restricting GAIA to a limiting magnitude of $15 \mathrm{mag}$, or to a subset of all objects down to its detection limit, would provide a reduction in the down-link telemetry rate, but little or no change in the other main aspects of the payload design. These are driven simply by the photon noise budget required to reach a $10 \mu$ as accuracy at $15 \mathrm{mag}$. The faint magnitude limit, the ability to meet the adopted scientific case, and the number of target objects follow from the accuracy requirement, with no additional spacecraft cost.

\subsection{Radial velocity measurements}

There is one dominant scientific requirement, as well as two additional scientific motivations, for the acquisition of radial velocities with GAIA: (i) astrometric measurements supply only two components of the space motion of the target stars: the third component, radial velocity, is directed along the line of sight, but is nevertheless essential for dynamical studies; (ii) measurement of the radial velocity at a number of epochs is a powerful method for detecting and characterising binary systems; (iii) at the GAIA accuracy levels, "perspective acceleration" is at the same time both a complication and an important observable quantity. If the distance between an object and observer changes with time due to a radial component of motion, a constant transverse velocity is observed as a varying transverse angular motion, the perspective acceleration. Although the effect is generally small, some hundreds of thousands of high-velocity stars will have systematic distance errors if the radial velocities are unknown.

On-board acquisition of radial velocities with GAIA is not only feasible, but is relatively simple, is scientifically necessary, and cannot be readily provided in any other way. In terms of accuracy requirements, faint and bright magnitude regimes can be distinguished. The faint targets will mostly be distant stars, which will be of interest as tracers of Galactic dynamics. The uncertainty in the tangential component of their space motion will be dominated by the error in the parallax. Hence a radial velocity accuracy of $\simeq 5 \mathrm{~km} \mathrm{~s}^{-1}$ is sufficient for statistical purposes. Stars with $V \lesssim 15$ mag will be of individual interest, and the radial velocity will be useful also as an indicator of multiplicity and for the determination of perspective acceleration. The radial velocities will be determined by digital cross-correlation between an observed spectrum and an appropriate template. The present design allows (for red Population I stars of any luminosity class) determination of radial velocities to $\sigma_{v} \simeq 5 \mathrm{~km} \mathrm{~s}^{-1}$ at $V=18 \mathrm{mag}$ (e.g. Munari 1999a).

Most stars are intrinsically red, and made even redder by interstellar absorption. Thus, a red spectral region is to be preferred for the GAIA spectrograph. To maximize the radial velocity signal even for metal-poor stars, strong, saturated lines are desirable. Specific studies, and groundbased experience, show that the Ca II triplet near $860 \mathrm{~nm}$ is optimal for radial velocity determination in the greatest number of stellar types.

Ground-based radial velocity surveys are approaching the one million-object level. That experience shows the cost and complexity of determining some hundreds of millions of radial velocities is impractical. There is also a substantial additional scientific return in acquiring a large number of measurements, and doing so not only well spaced in time but also, preferably, simultaneously with the astrometric measurements (e.g. variables and multiple systems).

\subsection{Derivation of astrophysical parameters}

The GAIA core science case requires measurement of luminosity, effective temperature, mass, age and composition, in addition to distance and velocity, to optimise understanding of the stellar populations in the Galaxy and its nearest neighbours. The quantities complementary to the kinematics can be derived from the spectral energy distribution of the stars by multi-band photometry and spectroscopy. Acquisition of this astrophysical information is an essential part of the GAIA payload. A broad-band magnitude, and its time dependence, will be obtained from the primary mission data, allowing both astrophysical analyses and the critical corrections for residual system chromaticity. For the brighter stars, the radial.

For essentially every application of the GAIA astrometric data, high-quality photometric data will be crucial, in providing the basic tools for classifying stars across the entire HR diagram, as well as in identifying specific and peculiar objects (e.g. Straizys 1999). Photometry must determine (i) temperature and reddening at least for OBA stars and (ii) effective temperatures and abundances for late-type giants and dwarfs. To be able to reconstruct Galactic formation history the distribution function of stellar abundances must be determined to $\sim 0.2 \mathrm{dex}$, while effective temperatures must be determined to $\sim 200 \mathrm{~K}$. Separate determination of the abundance of $\mathrm{Fe}$ and 

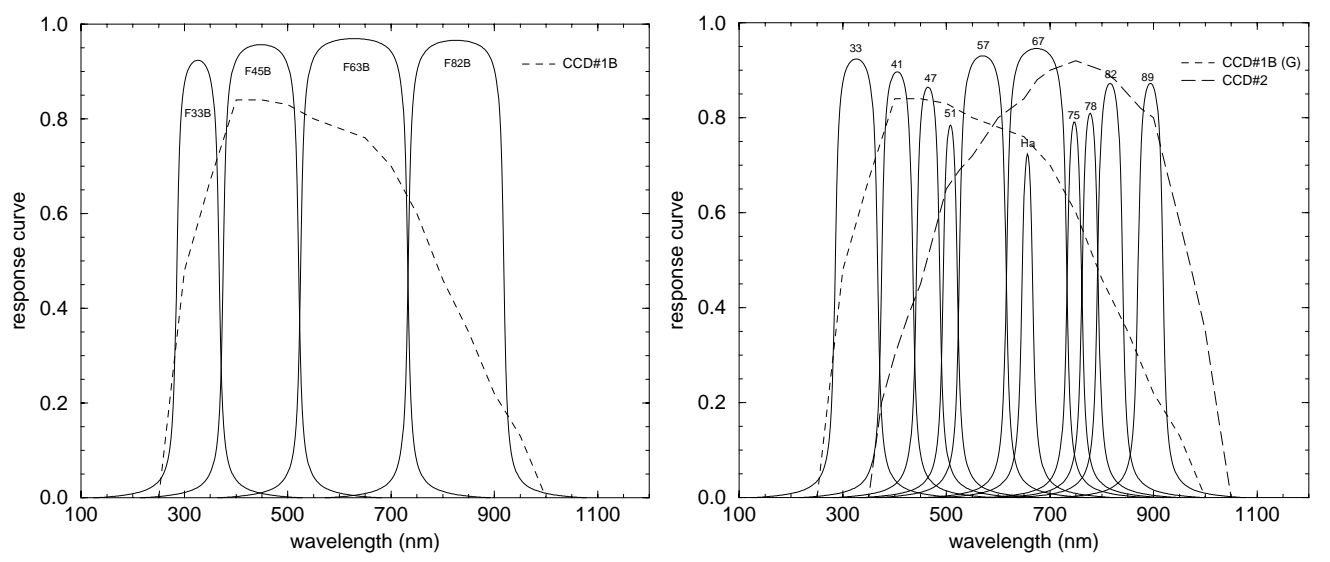

Fig. 1. Filter transmission curves and CCD response curves for the provisional (baseline) broad-band (left) and medium-band (right) photometric systems

$\alpha$-elements (at the same accuracy level) will be desirable for mapping Galactic chemical evolution. These requirements translate into a magnitude accuracy of $\simeq 0.02 \mathrm{mag}$ for each colour index.

Many photometric systems exist, but none is necessarily optimal for space implementation. For GAIA, photometry will be required for quasar and galaxy photometry, Solar System object classification, etc. Considerable effort has therefore been devoted to the design of an optimum filter system for GAIA (e.g. Høg et al. 1999a; Munari 1999b). The result of this effort is a baseline system, with four broad and eleven medium passbands, covering the near ultraviolet to the CCD red limit. The filters are summarised in Fig. 1. The 4 broad-band filters are implemented within the astrometric fields, and therefore yield photometry at the same angular resolution (also relevant for chromatic correction), while the 11 medium-band filters are implemented within the spectrometric telescope. Both target magnitude limits of $20 \mathrm{mag}$, as for the astrometric measurements.

\subsection{On-board detection}

Clear definition and understanding of the selection function used to decide which targets to observe is a crucial scientific issue, strongly driving the final scientific output of the mission. The optimum selection function, and that adopted, is to detect every target above some practical signal level on-board as it enters the focal plane. This has the advantage that the detection will be carried out in the same wave-band, and at the same angular resolution, as the final observations. The focal plane data on all objects down to about 20 mag can then be read out and telemetered to ground within system capabilities. All objects, including Solar System objects, variable objects, supernovae, and microlensed sources, are detected using this "astrometric sky mapper", described in further detail in Sect. 4.3.

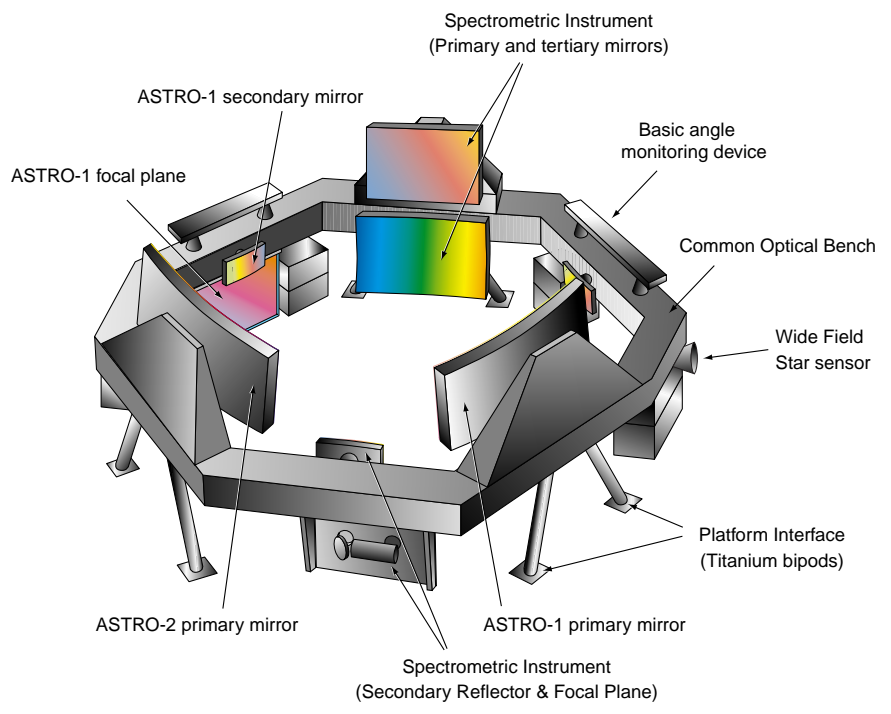

Fig. 2. The payload includes two identical astrometric instruments (labelled ASTRO-1 and ASTRO-2) separated by the $106^{\circ}$ basic angle, as well as a spectrometric instrument (comprising a radial velocity measurement instrument and a medium-band photometer) which share the focal plane of a third viewing direction. All telescopes are accommodated on a common optical bench of the same material, and a basic angle monitoring device tracks any variations in the relative viewing directions of the astrometric fields

\section{Payload design}

\subsection{Measurement principles}

The overall design constraints have been investigated in detail in order to optimise the number and optical design of each viewing direction, the choice of wavelength bands, detection systems, detector sampling strategies, basic angle, metrology system, satellite layout, and orbit (Mérat et al. 1999). The resulting proposed payload design (Fig. 2) consists of:

(a) two astrometric viewing directions. Each of these astrometric instruments comprises an all-reflective threemirror telescope with an aperture of $1.7 \times 0.7 \mathrm{~m}^{2}$, the two 
fields separated by a basic angle of $106^{\circ}$. Each astrometric field comprises an astrometric sky mapper, the astrometric field proper, and a broad-band photometer. Each sky mapper system provides an on-board capability for star detection and selection, and for the star position and satellite scan-speed measurement. The main focal plane assembly employs CCD technology, with about 250 CCDs and accompanying video chains per focal plane, a pixel size $9 \mu \mathrm{m}$ along scan, TDI (time-delayed integration) operation, and an integration time of $\sim 0.9$ s per CCD;

(b) an integrated radial velocity spectrometer and photometric instrument, comprising an all-reflective threemirror telescope of aperture $0.75 \times 0.70 \mathrm{~m}^{2}$. The field of view is separated into a dedicated sky mapper, the radial velocity spectrometer, and a medium-band photometer. Both instrument focal planes are based on CCD technology operating in TDI mode;

(c) the opto-mechanical-thermal assembly comprising: (i) a single structural torus supporting all mirrors and focal planes, employing $\mathrm{SiC}$ for both mirrors and structure. There is a symmetrical configuration for the two astrometric viewing directions, with the spectrometric telescope accommodated within the same structure, between the two astrometric viewing directions; (ii) a deployable Sun shield to avoid direct Sun illumination and rotating shadows on the payload module, combined with the Solar array assembly; (iii) control of the heat injection from the service module into the payload module, and control of the focal plane assembly power dissipation in order to provide an ultra-stable internal thermal environment; (iv) an alignment mechanism on the secondary mirror for each astrometric instrument, with micron-level positional accuracy and $200 \mu \mathrm{m}$ range, to correct for telescope aberration and mirror misalignment at the beginning of life; (v) a permanent monitoring of the basic angle, but without active control on board.

The accuracy goal is to reach a $10 \mu$ as rms positional accuracy for stars of magnitude $V=15 \mathrm{mag}$. For fainter magnitudes, the accuracy falls to about $20-40 \mu$ as at $V=17-18 \mathrm{mag}$, and to $100-200 \mu$ as at $V=20 \mathrm{mag}$, entirely due to photon statistics. For $V<15$ mag, higher accuracy is achieved, but will be limited by systematic effects at about $3-4 \mu$ as for $V<10-11$ mag. Raw data representing the star profile along scan must be sent to ground. An integral objective of the mission is to provide the sixth astrometric parameter, radial velocity, by measuring the Doppler shift of selected spectral lines. Colour information is to be acquired for all observed objects, primarily to allow astrophysical analyses, though calibration of the instrument's chromatic dependence is a key secondary consideration.

The astrometric accuracy can be separated into two independent terms, the random part induced by photoelectron statistics on the localisation process accuracy, and a bias error which is independent of the number of collected photons. The random part decreases in an ideal system as $N^{-0.5}$, where $N$ is the number of detected electrons per star; the bias part is independent of $N$,
Table 5. Summary of the scanning law and pointing requirements. $0.05 \mathrm{~Hz}$ is the maximum frequency that can be identified after measurement post-processing

\begin{tabular}{ll}
\hline Parameter & Value \\
\hline Satellite scan axis tilt angle & $55^{\circ}$ to the Sun \\
Scan rate & $120 \operatorname{arcsec~s}^{-1}$ \\
Absolute scan rate error & $1.2 \operatorname{arcsec~s}{ }^{-1}(3 \sigma)$ \\
Precession rate & $0.17 \operatorname{arcsec~s}{ }^{-1}$ \\
Absolute precession rate error & $0.1 \operatorname{arcsec~s}(3 \sigma)$ \\
Absolute pointing error & $5 \operatorname{arcmin}(3 \sigma)$ \\
Attitude absolute measurement error & $0.001 \operatorname{arcsec}(1 \sigma)$ \\
High-frequency disturbances: & \\
$\quad$ power spectral density at $0.05 \mathrm{~Hz}$ & $\leq 1000 \mu \operatorname{as}^{2} \mathrm{~Hz}^{-1}$ \\
$\quad$ for $f>0.05 \mathrm{~Hz}$ & decreasing as $f^{-2}$ \\
\hline
\end{tabular}

represents the ultimate capability of the system for bright stars, is limited by payload stability on timescales shorter than those which can be self-calibrated, i.e. shorter than about 5 hours.

GAIA will operate through continuous sky scanning, this mode being optimally suited for a global, survey-type mission with very many targets, and being of proven validity from Hipparcos. The satellite scans the sky according to a pre-defined pattern in which the axis of rotation (perpendicular to the three viewing directions) is kept at a nominally fixed angle $\xi$ from the Sun, describing a precessional motion about the Solar direction at constant speed with respect to the stars. This angle is optimised against satellite Sun shield demands, parallax accuracy, and scanning law. Resulting satellite pointing performances are determined from operational and scientific processing requirements on ground, and are summarised in Table 5.

A mission length of 5 years is adopted for the satellite design lifetime, which starts at launcher separation and includes the transfer phase and all provisions related to system, satellite or ground segment dead time or outage. A lifetime of 6 years has been used for the sizing of all consumables.

\subsection{Optical design}

The astrometric telescopes have a long focal length, necessary for oversampling the individual images. A pixel size of $9 \mu \mathrm{m}$ in the along-scan direction was selected, with the $50 \mathrm{~m}$ focal length allowing a 6-pixel sampling of the diffraction image along scan at $600 \mathrm{~nm}$. The resulting optical system is very compact, fitting into a volume $1.8 \mathrm{~m}$ high, and within a mechanical structure adapted to the Ariane 5 launcher. Deployable payload elements have been avoided. System optimisation yields a suitable full pupil of $1.7 \times 0.7 \mathrm{~m}^{2}$ area with a rectangular shape. Optical performances which have been optimised are the image quality, characterised by the wave-front error, and the along-scan distortion, avoiding at the same time a curved focal plane in order to facilitate CCD positioning and 
mechanical complexity. The optical configuration is derived from a three-mirror anastigmatic design with an intermediate image. The three mirrors have aspheric surfaces with limited high-order terms, and each of them is a part of a rotationally symmetric surface. The aperture shapes are rectangular and decentered, while each mirror is slightly tilted and decentered.

The tolerable optical distortion arises from the requirement that any variation of scale across the field must not cause significant image blurring during TDI operation. The number and size of the CCDs has been determined to match the optical quality locally in the field.

The monochromatic point spread function, $P_{\lambda}(\xi, \eta)$, at a specific point in the field, is related to the corresponding wavefront error map $w(x, y)$ in the pupil plane through the diffraction formula. The overall wavefront error of the telescope is the sum of the errors arising from optical design, alignment, and polishing residuals for the three mirrors. The design target of $\lambda / 50 \mathrm{rms}$ over the whole field corresponds to a Strehl ratio of 0.84 at $500 \mathrm{~nm}$. From analysis performed using the optical design software package Code V, alignment errors can be made negligible (wave front error $<\lambda / 70 \mathrm{rms}$ ) provided that the mirrors are positioned with an accuracy of about $\pm 1 \mu \mathrm{m}$. A 5 degreeof-freedom compensation mechanism with this accuracy (not considered to be excessively stringent with piezo-type actuators) is therefore implemented on the secondary reflector of each of the astrometric telescopes. This allows optimization of the overall optical quality in orbit as a result of on-ground residual alignment errors, and the recovery of misalignments of the telescope optics which may be induced by launch effects, even if all the mirrors are randomly misaligned by an amplitude $\pm 50 \mu \mathrm{m}$ in all directions. The required wavefront error measurement will be performed on at least three points of the field of view. In practice, the astrometric performance is not strongly dependent on the actual telescope wavefront error, since the effect of aberrations corresponds to first order in an energy loss in the central diffraction peak, which is the only part of the point spread function used for the star localization.

Although the optical design only employs mirrors, diffraction effects with residual (achromatic) aberrations induce a small chromatic shift of the diffraction peak. The chromaticity image displacement depends on position in the field, and on the star's spectral energy distribution (colour), but not on its magnitude. One purpose of the broad-band photometric measurements within the main field is to provide colour information on each observed object in the astrometric field to enable this chromaticity bias calibration on ground. Recent developments made on ion beam polishing have shown that polishing errors can be made practically negligible $(\lambda / 100 \mathrm{rms}$ obtained on a $\mathrm{SiC}$ reflector of about $200 \mathrm{~mm}$ diameter). It is therefore likely that the chromatic shift can be reduced below a few tens of $\mu$ as over the whole field, easing calibration requirements. Combination of the satellite Sun shield and internal baffling reduce straylight to negligible levels.

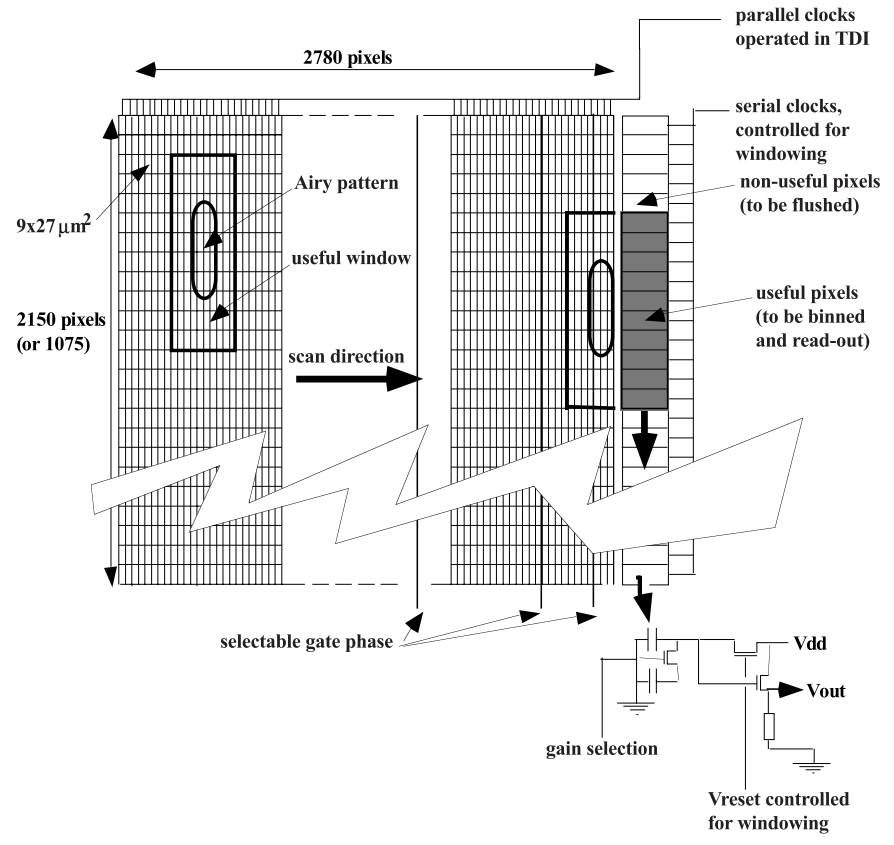

Fig. 3. Operating mode for the astrometric field CCDs. The location of the star is known from the astrometric sky mapper, combined with the satellite attitude. A window is selected around the star in order to minimise the resulting read-out noise of the relevant pixels

\subsection{Astrometric focal plane}

The focal plane contains a set of CCDs operating in TDI (time-delayed integration) mode, scanning at the same velocity as the spacecraft scanning velocity and thus integrating the stellar images until they are transferred to the serial register for read out. Three functions are assigned to the focal plane system: (i) the astrometric sky mapper; (ii) the astrometric field, devoted to the astrometric measurements; (iii) the broad band photometer, which provides broad-band photometric measurements for each object. The same elementary CCD is used for the entire focal plane, with minor differences in the operating modes depending on the assigned functions.

The astrometric sky mapper detects objects entering the field of view, and communicates details of the star transit to the subsequent astrometric and broad-band photometric fields. Three CCD strips provide (sequentially) a detection region for bright stars, a region which is read out completely to detect all objects crossing the field, and a third region which reads out detected objects in a windowed mode, to reduce read-out noise (and hence to improve the signal-to-noise ratio of the detection process), and to confirm objects provisionally detected in the previous CCD strips, in the presence of, e.g., cosmic rays. Simulations have shown that algorithms such as those developed for the analysis of crowded photometric fields (e.g. Irwin 1985) can be adapted to the problem of on-board detection, yielding good detection probabilities to $20 \mathrm{mag}$, with low spurious detection rates. Passages of stars across the sky mapper yield the instantaneous satellite spin rate, 


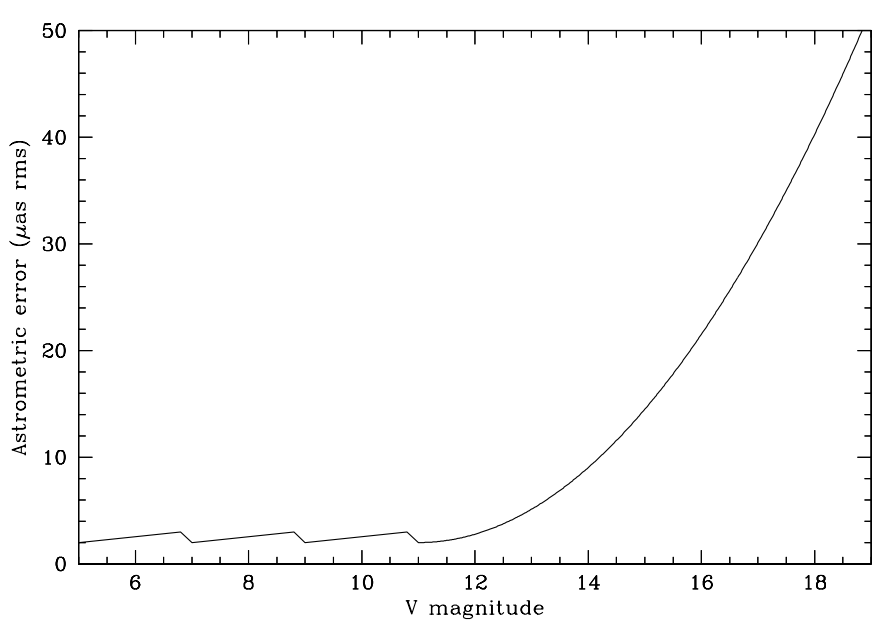

Fig. 4. Nominal accuracy performance versus magnitude, for a G2V star. For $V<15$ mag the astrometric performance improves because the number of detected photons increases until the detector saturation level is reached $(V \sim 11.6$ for $\mathrm{G} 2 \mathrm{~V}$ star). Brighter than this, the performance is practically independent of magnitude, due to the pre-selection of the number of TDI stages required to avoid saturation

and allow the prediction of the the individual star transits across the main astrometric field with adequate precision for the foreseen windowing mode.

The size of the astrometric field is optimised at system level to achieve the specified accuracy, with a field of $0.5 \times 0.66$. The size of the individual CCD is a compromise between manufacturing yield, distortion, and integration time constraints. The pixel size is a compromise between manufacturing feasibility, detection performances (QE and MTF), and charge-handling capacity: a dimension of $9 \mu \mathrm{m}$ in the along-scan direction provides full sampling of the diffraction image, and a size of $27 \mu \mathrm{m}$ in the across-scan direction is compatible with the size of the dimensions of the point spread function and cross scan image motion. In addition, it provides space for implementation of special features for the CCD (e.g. pixel anti-blooming drain) and provides improved chargehandling capacity. Quantitative calculations have demonstrated that the pixel size, TDI smearing, pixel sampling, and point spread function are all matched to system requirements. The CCDs are slightly rotated in the focal plane and are individually sequenced in order to compensate for the telescope optical distortion. Cross-scan binning of 8 pixels is implemented in the serial register for improvement of the signal-to-noise ratio.

Each individual CCD features specific architecture allowing measurement of stars brighter than the normal saturation limit of about $V=11-12$ mag: selectable gate phases allow pre-selection of the number of TDI stages to be used within a given CCD array. The resulting astrometric error versus magnitude shows the effect of this discrete selection (Fig. 4).

At the apparent magnitude and integration time limits appropriate for GAIA most of the pixel data do not include any useful information. There is a clear trade-off between reading too many pixels, with associated higher read-noise and telemetry costs, and reading too few, with associated lost science costs. This contributes to the choice of on-board real-time detection, with definition of a window around each source which has sufficient signal to be studiable, and determination of the effective sensitivity limit to be that which saturates the telemetry, and which provides a viable lower signal. Combining all these constraints sets the limit near $V=20$ mag, resulting in an estimated number of somewhat over one billion targets.

The broad-band photometric field provides multicolour, multi-epoch photometric measurements for each object observed in the astrometric field, for chromatic correction and astrophysical analysis. Four photometric bands are implemented within each instrument.

\subsection{Spectrometric instrument}

A dedicated telescope, with a rectangular entrance pupil of $0.75 \times 0.70 \mathrm{~m}^{2}$, feeds both the radial velocity spectrometer and the medium-band photometer: the overall field of view is split into a central $1^{\circ} \times 1^{\circ}$ devoted to the radial velocity measurements, and two outer $1^{\circ} \times 1^{\circ}$ regions devoted to medium-band photometry. The telescope is a 3 -mirror standard anastigmatic of focal length $4.17 \mathrm{~m}$. The mirror surfaces are coaxial conics. An all-reflective design allows a wide spectral bandwidth for photometry. The image quality at telescope focus allows the use of $10 \times 10 \mu \mathrm{m}^{2}$ pixels within the photometric field, corresponding to a spatial resolution of 0.5 arcsec.

The radial velocity spectrometer acquires spectra of all sufficiently bright sources, and is based on a slitless spectrograph comprising a collimator, transmission grating plus prism (allowing TDI operation over the entire field of view) and an imager, working at unit magnification. The two lens assemblies (collimating and focusing) are identical, compensating odd aberrations including coma and distortion. The dispersion direction is perpendicular to scan direction. The overall optical layout is shown in Fig. 5. The array covers a field height of $1^{\circ}$. Each $20 \times 20 \mu \mathrm{m}^{2}$ pixel corresponds to an angular sampling of 1 arcsec and a spectral sampling of approximately $0.075 \mathrm{~nm} /$ pixel. The focal plane consists of three CCDs mechanically butted together, each operated in TDI mode with its own sequencing, providing read-noise as low as $3 \mathrm{e}^{-}$rms with the use of a dedicated "skipper-type" multiple non-destructive readout architecture with 4 nondestructive readout samples per pixel.

The requirements for the CCDs are very similar to those of the astrometric field, including the use of TDI, and dedicated sky mapper. The photometric bands (Fig. 1) will require filters to be directly fixed onto the CCD array. 

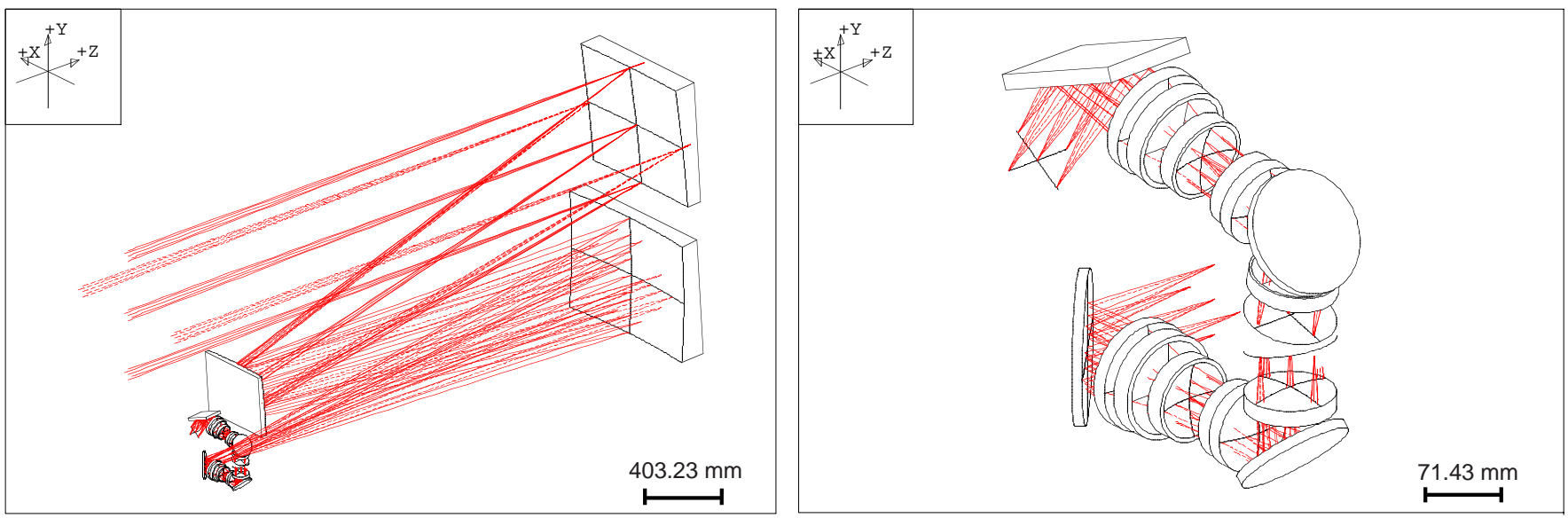

Fig. 5. Optical configuration of the spectrometric instrument. The left figure shows the overall telescope design, while the right figure shows details of the spectrograph optics

\subsection{Science data acquisition and on-board handling}

Preliminary investigations have been carried out to identify the minimum set of data to be transmitted to ground to satisfy the scientific mission objectives; to identify some on-board data discrimination compression principles able to provide the targeted data compression ratio; to assess the feasibility and complexity of implementing such compression strategies and related algorithms on board; to assess the resulting compressed data rate at payload output, which are used for the sizing of the solid state memory and communication subsystem; and to derive preliminary mass, size, and power budgets for the on-board processing hardware. For estimating telemetry rates (Table 6), a specific spatial sampling of the CCD data has been assumed. This sampling is not yet optimised and final, but represents a useful first approximation.

The instantaneous data rate will primarily fluctuate with the stellar density in each of the three fields of view, which scale with Galactic latitude. On-board storage will store a full day of observation for downlink at a higher rate during ground-station visibility. Including overhead, the total raw science data rate is roughly a factor 7 higher than the mean (continuous) payload data rate foreseen in

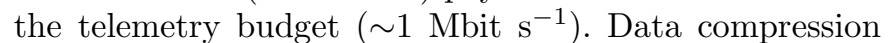
will reduce this discrepancy, but there remains roughly a factor two to be gained either by smarter CCD sampling, or by increasing the link capacity.

\section{6. $C C D$ details}

CCD detectors form the core of the GAIA payload: their development and manufacture represents one of the key challenges for the programme. In the present study, consideration was given to the requirements on electro-optical behaviour; array size; buttability; pixel size; bright star handling; serial register performance; output amplifiers; power dissipation in the image zone, serial register, and output amplifier; trade-off between QE and MTF; photoresponse non-uniformity; dark current; conversion factor and linearity; charge handling capacity per pixel; charge transfer efficiency in the image zone and serial register; minimization of residual images; anti-blooming efficiency; and packaging. The present baseline design is summarised in Table 7.

For astrometric use CCD accuracy depends essentially on the integral of $\mathrm{QE} \times \mathrm{MTF}$ over the wavelength band. CCD MTF must be optimised in parallel with the QE. The QE and MTF values for the CCD optimised for the astrometric field have been used in the detailed astrometric accuracy analysis. The pixel size, nominally adopted as $9 \times 27 \mu \mathrm{m}^{2}$ for the astrometric field, is an important design parameter. A smaller pixel size would decrease the telescope focal length, as well as the overall size of the astrometric focal plane assembly, and consequently the overall size of the overall payload. However, such devices provide worse performance in a number of other areas. The trade-off between QE, MTF, and charge-handling capacity results in design reference values, and bread-boarding activities are underway to verify these performances in detail.

A "worst-case" star density, corresponding to about $2.810^{6}$ stars per square degree (about 19-20 mag in Baade's Window) has been used in a detailed analysis of CCD performance. The total noise per sample includes contributions from the CCD read-out noise at the relevant read frequency, analog-to-digital conversion noise, and the video chain analog noise.

All CCDs used for both astrometric and photometric measurements are operated in the drift-scan or TDI (time delay and integration) mode. That is, charge packets are gradually built up while transferred from pixel to pixel at the same rate as the optical image moves across the detector. Centroiding on the digitised output provides a measure of the position of the optical image relative to the electronic transfer along the pixel columns. Demands on the precision of the mean image position are strict: a standard error of $10 \mu$ as in the final trigonometric parallax translates to a required centroiding precision in the astrometric field of $36 \mathrm{~nm}, 1 / 250$ of a pixel. 
Table 6. Average stellar flow in the various fields of the astrometric and spectrometric instruments, and the resulting average telemetry rates. A limiting of $G=20$ mag is assumed for the astrometric instrument (AF and BBP) and for the medium-band photometer (MBP), and $G=17$ mag for the radial-velocity spectrometer (RVS). It is assumed that each sample represents 16 bits of raw data. The resulting raw data rates are before compression and do not include overhead

\begin{tabular}{|c|c|c|c|c|c|c|c|}
\hline \multirow[t]{2}{*}{ Parameter } & \multicolumn{4}{|c|}{ Astro-1 and 2 (per instrument) } & \multicolumn{3}{|c|}{ Spectro } \\
\hline & ASM3 & AF01-16 & AF17 & $\mathrm{BBP}$ & SSM1 & MBP & RVS \\
\hline Limiting magnitude, $G_{\max }[\mathrm{mag}]$ & \multicolumn{4}{|c|}{20} & \multicolumn{2}{|c|}{20} & 17 \\
\hline Average star density, $N_{\mathrm{s}}\left[\mathrm{deg}^{-2}\right]$ & \multicolumn{4}{|c|}{25000} & \multicolumn{2}{|c|}{25000} & 2900 \\
\hline TDI integration time per CCD, $\tau_{1}[\mathrm{~s}]$ & \multicolumn{4}{|c|}{0.86} & \multicolumn{2}{|c|}{3.0} & 30 \\
\hline Field width across scan, $\Phi_{y}[\mathrm{deg}]$ & \multicolumn{4}{|c|}{0.66} & \multicolumn{2}{|c|}{1.0} & 1.0 \\
\hline Star flow through $\Phi_{y}, f=N_{\mathrm{s}} \Phi_{y} \omega\left[\mathrm{s}^{-1}\right]$ & \multicolumn{4}{|c|}{550} & \multicolumn{2}{|c|}{833} & 97 \\
\hline Number of CCDs along scan, $N_{\mathrm{CCD}}$ & 1 & 16 & 1 & 4 & 1 & 14 & 1 \\
\hline Solid angle of CCDs, $\Omega\left[\mathrm{deg}^{2}\right]$ & 0.019 & 0.302 & 0.019 & 0.077 & 0.100 & 1.400 & 1.000 \\
\hline Number of stars on the CCDs, $N_{\mathrm{s}} \Omega$ & 473 & 7568 & 473 & 1892 & 2500 & 35000 & 2900 \\
\hline Readout rate, $R=N_{\mathrm{s}} \Omega / \tau_{1}\left[\mathrm{~s}^{-1}\right]$ & 550 & 8800 & 550 & 2200 & 833 & 11662 & 97 \\
\hline Samples per star read out & 25 & 6 & 30 & 16 or 10 & 42 & 14 & 930 \\
\hline Samples per star transmitted, $n$ & 25 & 6 & 30 & 10 & 42 & 8 & 930 \\
\hline Raw data rate, $16 n R\left[\mathrm{kbit} \mathrm{s}^{-1}\right]$ & 220 & 845 & 264 & 352 & 560 & 1494 & 1443 \\
\hline Raw data rate per instrument $\left[\mathrm{kbit} \mathrm{s}^{-1}\right]$ & \multicolumn{4}{|c|}{1681} & \multicolumn{3}{|c|}{3496} \\
\hline Total raw data rate $\left[\mathrm{kbit} \mathrm{s}^{-1}\right]$ & \multicolumn{7}{|c|}{$2 \times 1681+3496=6858$} \\
\hline
\end{tabular}

Specific laboratory experiments, using the $13 \mu \mathrm{m}$ pixel EEV device CCD42-10 in windowing mode, non-irradiated as well as irradiated at doses of up to $510^{9}$ protons $\mathrm{cm}^{-2}$, have been conducted in TDI mode, using different illumination levels, and at different CCD operating temperatures. Although not fully representative of the flight configuration, and while not yet fully evaluated, these experiments have demonstrated that the targetted centroiding accuracy appears to be achievable.

A key parameter for achieving a high degree of reproducibility is the Charge Transfer Efficiency (CTE) of the CCD. In the present context it is more convenient to discuss the Charge Transfer Inefficiency (CTI) $\varepsilon=1-$ CTE. Very few charge carriers are actually lost (through recombination) during the transfer process; rather, some carriers are captured by "traps" and re-emitted at a later time, thus ending up in the "wrong" charge packet at the output; if short-time constant processes dominate, the main effect observed is that of image smearing. The CTI has an effect both on the photometric measurement (by reducing the total charge remaining within the image) and the astrometric measurement (by shifting charges systematically in one direction). CTI during parallel transfer is particularly critical, since it affects the astrometric measurements in the direction where the highest precision is required, i.e. along the scan. In addition, CTI is worse along-scan due to the lower transfer rate. The magnitude of the problem can be crudely estimated as follows: assume a constant fraction $\varepsilon$ of the charge is left behind while the fraction $1-\varepsilon$ flows into the next pixel. The expected centroid shift is $\simeq N \varepsilon / 2$ pixels. The CCDs in the astrometric field of GAIA have $N=2780$ pixels of size $9 \mu \mathrm{m}$ along the scan.
Assuming $\varepsilon=10^{-5}$ results in a centroid shift of $125 \mathrm{~nm}$ or about $500 \mu$ as.

More careful appraisal of the CTI effects on the astrometric accuracy show the effect after calibration is negligible for the the undamaged (beginning-of-life) CCD, but potentially serious for the degraded performance that may result after significant exposure to particle radiation in orbit. Although most of the CTI effects can be calibrated as part of the normal data analysis, stochastic effects related to the charge losses can never be eliminated by clever processing. Extensive laboratory experiments are underway to quantify the amplitude of these residual effects.

\subsection{Payload summary}

In summary, the GAIA payload comprises the following elements:

(a) two identical astrometric telescopes:

- fully-reflective 3-mirror SiC optics,

- separation of viewing directions: $106^{\circ}$,

- monolithic primary mirrors: $1.7 \times 0.7 \mathrm{~m}^{2}$,

- field of view: $0.32 \mathrm{deg}^{2}$,

- focal length: $50 \mathrm{~m}$,

- wavelength range: $300-1000 \mathrm{~nm}$,

- 4-colour broad-band photometry,

- operating temperature: $\sim 200 \mathrm{~K}$,

- detectors: CCDs operating in TDI mode,

- pixel size along-scan: $9 \mu \mathrm{m}$; 
Table 7. Summary properties of the GAIA CCDs in the two astrometric telescopes $(\mathrm{QE}=$ quantum efficiency; $\mathrm{MTF}=$ modulation transfer function; CTI $=$ charge transfer inefficiency; RON = read-out noise)

\begin{tabular}{|c|c|}
\hline Feature & Details \\
\hline Array size & $25 \times 58 \mathrm{~mm}^{2}$ active area \\
\hline Pixels per CCD & 2150 cols $\times 2780$ TDI stages \\
\hline Dead zones & $\begin{array}{l}\text { top: } 0.25 \mathrm{~mm} \text {; sides: } 0.6 \mathrm{~mm} \text {; } \\
\text { bottom: }<5 \mathrm{~mm}\end{array}$ \\
\hline Pixel size in image zone & $9 \times 27 \mu \mathrm{m}^{2}$ \\
\hline Phases in image zone & 4 \\
\hline Pixel size in serial register & $27 \times 27 \mu \mathrm{m}^{2}$ \\
\hline Phases in serial register & 4 \\
\hline Device thickness & $10-12 \mu \mathrm{m}$ \\
\hline Si resistivity & $20-100 \Omega \mathrm{cm}$ \\
\hline Buried channel & n-type channel \\
\hline Oxide thickness & standard \\
\hline Anti-blooming & shielded at pixel level \\
\hline Notch channel & implanted for all CCDs \\
\hline Output amplifiers & 2 per device, 2 -stage \\
\hline Conversion factor & between $3-6 \mu \mathrm{V} / \mathrm{e}^{-}$ \\
\hline Additional gates & $5-10$ \\
\hline Power dissipation & $<560 \mathrm{~mW}$ \\
\hline Non-uniformity & $<1 \%$ rms local \\
\hline & $<10 \%$ peak-to-peak global \\
\hline Mean dark current & $<0.5 \mathrm{e}^{-} \mathrm{s}^{-1} \mathrm{pix}^{-1}(200 \mathrm{~K})$ \\
\hline Non-linearity & $\begin{array}{l}<1 \text { per cent over } 0-2 \mathrm{~V} ; \\
<20 \text { per cent over } 2-3.5 \mathrm{~V}\end{array}$ \\
\hline CTI in image area & $\begin{array}{l}<10^{-5} \text { at beginning-of-life; } \\
\sim 10^{-4} \text { after major Solar flare }\end{array}$ \\
\hline CTI in serial register & $\begin{array}{l}<10^{-5} \text { at beginning-of-life; } \\
\sim 5.10^{-4} \text { after major flare }\end{array}$ \\
\hline Quantum efficiency & trade with MTF and RON \\
\hline MTF at Nyquist frequency & trade with QE and RON \\
\hline Read-out noise & trade with QE and MTF \\
\hline
\end{tabular}

(b) spectrometric instrument:

- spectrometer for radial velocities,

- 11 colour medium-band photometry,

- entrance pupil: $0.75 \times 0.70 \mathrm{~m}^{2}$,

- field of view: $4 \mathrm{deg}^{2}$,

- focal length: $4.17 \mathrm{~m}$,

- detectors: CCDs operating in TDI mode.

\section{Spacecraft system}

\subsection{Design and operation}

The spacecraft subsystems provide all necessary support to the payload instrumentation. Designs follow well-established spacecraft engineering approaches, with innovative features within the mechanical, thermal, and telecommunication subsystems. Viable designs have been developed for the mechanical structure, thermal control, propulsion and attitude control, payload data handling, power and electrical subsystems, and communications.

The payload mechanical and thermal design provides the required stability passively. The stable orbit, combined with the Sun shield thermal cover and the constant Solar aspect angle, minimise external perturbations. The payload material should have a low coefficient of thermal expansion; high thermal conductivity to variable heat loads; suitable structural and optical properties; and a good light-weighting capability. Silicon carbide, as planned for FIRST, Rosetta, and SOFIA, appears to be the optimum material. The properties of silicon carbide allow it to be used both for the telescope mirrors and the payload torus structure, providing a homogeneous, high conductivity, athermal payload. The monolithic mirror sizes are compatible with present manufacturing capability.

An open-back torus will support the optical instruments and focal plane, and electronic units, ensuring the optical alignment is insensitive to uniform temperature variations.

The required line-of-sight stability (1 $\mu$ as rms) is high, while the short-term basic angle stability over the satellite revolution period ( 3 hours) is the only critical parameter so far identified which cannot be properly calibrated by on-ground data processing. Detailed thermal/mechanical analyses show that a basic angle variation of $1 \mu$ as rms corresponds to thermal gradient variations of $\sim 25 \mu \mathrm{K}$ in the payload module torus structure, and corresponding displacements in the range of $20-40 \mathrm{pm}$. This motion need not be controlled, but must be measured. A measurement device has been designed, and proven in an industrial contract (TNO/TPD Delft). This real measurement proved the viability of the basic angle measurement technique, and additionally demonstrated that a pattern on the CCD can be localized with an accuracy compatible with the mission requirements.

The Sun shield is a simple, but large, multi-layer insulation disk, providing thermal stability for the payload module, integrated with the Solar panels.

The payload module opto-mechanical stability is sensitive to residual thermal variations. The thermal control principle is based on specific regulation and insulation for each thermal source, exploiting the natural long time constant of the thermal cavity. The service module thermal control design objectives are to maintain electronic units inside the temperature specifications, and to avoid Sun reflections, hot spots or turning shadows on the payload module cavity towards Sun shield and service module interface. This is achieved, with reliance on the multi-layer insulation Sun shield. 


\subsection{Attitude and orbit control}

The total $\Delta V$ budget required for injection into and maintenance of the L2 operational orbit, including attitude control, has been quantified. Transition between the transfer orbit and the operational phase at L2 has been identified as one of the design drivers of the attitude control subsystem. The attitude control measurement subsystem utilises a mix of star sensors, a Sun acquisition sensor, payload instrument sky-mappers, a gyroscope (not used in operational mode), an attitude anomaly detector (for safe mode), bi-propellant thrusters, and FEEP (field-effect electric propulsion) thrusters to control satellite motion. The solution with a star sensor has been adopted since it provides simplification at instrument level, at system level, and at mission level. A detailed evaluation of the relative merits of using spin-stabilisation or 3-axis stabilisation during the transfer phase has been carried out, taking into account the overall system complexity, the required thruster configuration, and the necessary propellant consumption. The spin-stabilisation concept is adopted as baseline.

External perturbing sources are those induced by the Sun and the gravitational effects of nearby planets. The latter effect concerns only orbit drift. Solar pressures are created primarily by Solar radiation and by the Solar wind. The Solar pressure is subject to low-frequency variations which are modeled by an incoherent noise superimposed on the (seismological) 5 min oscillations.

The total spacecraft propellant budget is summarised as follows: (i) orbit correction: $983 \mathrm{~kg}$ of propellant are required; that is about one third of the launch mass, and can be considerably reduced (to typically $200 \mathrm{~kg}$ ) if the launcher is able to directly inject the spacecraft on the transfer trajectory to L2 (restartable Ariane 5); (ii) transfer phase manoeuvres: $24 \mathrm{~kg}$; (iii) attitude control during operational phase: the Caesium propellant budget for the FEEP thrusters in order to compensate for the Sun radiation pressure disturbing torques is $0.1 \mathrm{~kg}$ over 6 years; (iv) orbit maintenance during operational phase: $1.6 \mathrm{~kg}$ of Caesium propellant. In conclusion, the total propellant budget for the bi-propellant system is $1007 \mathrm{~kg}$ (including 10 percent margin) and $2.7 \mathrm{~kg}$ for the FEEP-based system (including 50 percent margin).

\subsection{Electrical, power and telemetry}

The spacecraft science data electrical architecture is complex, and has several critical tasks. There is one focal plane array per astrometric instrument, one for the radial velocity spectrometer and one for the medium-band photometer. Each focal plane array includes CCD arrays and front end electronics. The latter includes the video preamplifiers as well as all the necessary bias and voltage filters and clock drivers which have to be implemented close to the detectors. The focal plane array of each astrometric instrument includes about $250 \mathrm{CCDs}$, corresponding to about 300 video chains, has a mass of $40 \mathrm{~kg}$, a power dissipation of $170 \mathrm{~W}$, and approximately 2500 interface cables with the video processing unit. The detailed architecture of these focal plane arrays is a major issue which requires further investigations.

The video processing units include all the video chains up to the digitisation stage, the data discrimination function (star detection and discrimination by mean of a fixed programmable threshold), the localisation and datation of the detected events, the measurement of the scan rate (data extracted from the sky mapper field) and the transmission of these data to the spacecraft central computer for attitude control purposes, the multiplexing of the data and their transmission to the data handling and processing unit. Assuming use of hybrids and ASICs to improve the level of integration while minimising the power dissipation, four video processing unit boxes per astrometric focal plane are necessary for the hardware accommodation. The other main items, the payload data handling unit, a 100 Gbit solid-state recorder, and a high-rate telemetry formatter are not critical items.

A centralised electrical architecture is proposed, in which a single computer (the central data management unit) provides all the necessary control to all service module units, and the required command and control signals to the payload electronics. This minimisation of the quantity of hardware and software corresponds to a general trend in the design of modern platforms, and is realistic given the only moderate complexity of the thermal control and attitude and pointing control functions. This system, which is compatible with extant equipment, integrates a power subsystem, thermal control hardware, the reaction control subsystem, the attitude control measurement subsystem, and the communications subsystems.

The preliminary power budget has been computed in observational mode and with the spacecraft within ground station visibility, with all instruments and payload units switched on, and with the science telemetry down-link subsystem transmitting data to the ground station. The bottom-line requirement is $641 \mathrm{~W}$ service module, $1527 \mathrm{~W}$ payload, and $300 \mathrm{~W}$ contingency, for a total of $2468 \mathrm{~W}$. Adding interface losses to the spacecraft requirements, and considering aging effects, a total Ga-As Solar array surface of $24.1 \mathrm{~m}^{2}$ is required.

A feasible technical solution has been identified for the communication subsystem which is able to transmit the few Mbps required for the science data based on a single ground station concept. The Perth ground station presently offers the best compromise between coverage and performance and is considered as the baseline. There is no critical area identified at this stage for the telemetry and telecommand link. Omni-directional coverage will be provided by the on-board antennae, to cope with any spacecraft attitude at any time from launch through to the end of the mission. Relatively high RF transmitted power is required $(17 \mathrm{~W})$ in order to provide the required recovery margins from the L2 orbit. Following the standard approach for high-rate telemetry subsystems, this will require an additional solid-state power 
amplifier at the transponder output. Several technology candidates for the science telemetry link antenna have been identified and compared during the study: single beam fixed antenna, switched antenna network, electronically scanned phased array antenna, and single beam steerable antenna. Detailed studies have led to the adoption of an electronically scanned phased array (or "conformal") antenna as baseline, with a single beam steerable antenna as back-up solution. The phased array has no moving parts, which means that there is no source of dynamic perturbation for the attitude control and measurement subsystem. Although based on off-the-shelf technologies at elementary level, it will require significant engineering and development activities at assembly level, dedicated design and arrangement of the radiating elements, and the overall mechanical and thermal design.

\subsection{Orbit, operations, and ground segment}

Detailed assesment of orbit options indicate that a Lissajous orbit around the Earth-Sun Lagrange point L2 is the preferred option. The L2 region provides a very stable thermal environment (in order to satisfy the stringent geometrical stability requirements of the optical payload); an absence or minimisation of eclipses (which would perturb the thermal environment and geometrical stability of the payload, and thus require a more complex power subsystem and more complex satellite operation); an absence of Earth or Moon occultations (which would introduce straylight, thermal fluctuations, or blooming at detector level); more stable perturbing torques (dominated by Solar radiation pressure); and a lower radiation environment. The orbit is compatible with an Ariane 5 dual launch, and results in the lowest cost impact at system level, i.e. taking into account launch cost, induced satellite complexity, and operations cost. The orbit is consistent with a 6 -year extended lifetime, with minimum mission outages due to the transfer phase, perturbations, eclipses, etc.

The launch strategy selected as baseline is based on a dual or multiple launch with Ariane 5, followed by injection of the satellite from the standard geostationary transfer orbit into the L2 transfer orbit via an autonomous propulsion system. An alternative strategy using a "restartable" Ariane 5, as foreseen for Rosetta and for FIRST/Planck, would allow direct injection into the transfer orbit to L2, and substantially reduce launch mass.

Orbits around the co-linear libration point L2. (1.5 $10^{6} \mathrm{~km}$ from the Earth away from the Sun) are planned for NGST, FIRST/Planck, and GAIA. Detailed analysis has selected for GAIA a family of orbits around L2 which seen from the Earth describe a Lissajous figure, have a time span from eclipse to eclipse of about 6 years, require minimal injection $\Delta V$, and retain the Sunspacecraft-Earth angle below $15^{\circ}$.

The astrometric analysis requires a priori knowledge of the position and velocity vector of the satellite with respect to the Solar System barycentre. For positional precision, in the most demanding case, taking $\pi=1 \operatorname{arcsec}$ and $\sigma_{\pi}=10$ microarcsec, the satellite-barycentre distance needs to be known at the level of $10^{-6} \mathrm{AU}=150 \mathrm{~km}$. The Earth orbit is expected to be known with a relative accuracy of $10^{-10}$ in the near future. For velocity precision, the size of the correction is of the order of $v / c$, where $v$ is the barycentric velocity of the observer and $c$ the velocity of light. In order to compute the correction to within $\delta \alpha=1$ microarcsec $\left(510^{-12} \mathrm{rad}\right)$ the satellite barycentric velocity vector is needed to within $c \delta \alpha \sim 1.5 \mathrm{~mm} \mathrm{~s}^{-1}$. A prediction accuracy somewhat better than $0.1 \mathrm{~mm} \mathrm{~s}^{-1}$ is realistic within a few years.

The radiation environment presents a hazard to space systems, in addition to the effects on the focal plane detectors. Penetrating particles can induce upsets to electronics, payload interference, damage to components and deep dielectric charging. Outside of possible Solar flares, L2 predictions of cosmic ray particles have been derived using the well-established (CREME96) models. ISO and HST experience is being applied in shielding specification, although the total HST cosmic ray count rates are higher than for GAIA, which at L2 is well out of Earth's magnetosphere.

\subsection{Spacecraft summary}

The combined payload and service module results in a current total mass of $1696 \mathrm{~kg}$. Including a system margin of 20 percent results in a spacecraft dry mass of $2035 \mathrm{~kg}$. The total satellite mass depends significantly on whether a liquid apogee engine is required (total launch mass $3137 \mathrm{~kg}$ ), or whether the projected re-startable Ariane 5 capability allows this to be omitted (total launch mass $2267 \mathrm{~kg}$ ).

In summary, the spacecraft and orbit are characterised as follows:

- orbit: Lissajous-type, eclipse-free, around L2 point of Sun/Earth system; 220-240 day transfer orbit;

- sky scanning: revolving scanning with scan rate = $120 \operatorname{arcsec~}^{-1}$, precession period $=76$ days;

- spacecraft: 3-axis stabilized; autonomous propulsion system for transfer orbit; electrical (FEEP) thrusters for operational attitude control; 6 deployable Solar panels, integrated with multi-layer insulation to form the Sun shield;

- science data rate: 1 Mbps sustained, 3 Mbps on downlink, using electronically steerable high-gain phased array antenna;

- launch mass: $3137 \mathrm{~kg}$ (payload $=803 \mathrm{~kg}$, service module $=893 \mathrm{~kg}$, system margin $(20 \%)=339 \mathrm{~kg}$, fuel $=$ $1010 \mathrm{~kg}$, launch adaptor $=92 \mathrm{~kg}$ );

- power: $2569 \mathrm{~W}$ (payload $=1528 \mathrm{~W}$, service module $=$ $641 \mathrm{~W}$, harness losses $=76 \mathrm{~W}$, contingency $(10 \%)=$ $224 \mathrm{~W})$;

- payload dimensions: diameter $=4.2 \mathrm{~m}$, height $=2.1 \mathrm{~m}$;

- service module dimensions: diameter $=4.2 \mathrm{~m}$ (stowed) $/ 8.5 \mathrm{~m}$ (deployed), height $=0.8 \mathrm{~m}$;

- launcher: Ariane 5, dual launch; 
- lifetime: 5 years design lifetime (4 years observation time); 6 years extended lifetime.

\section{Accuracy assessment}

One important objective of the design studies has been to assess the overall performance of GAIA in relation to its scientific goals. This included the astrometric, photometric and radial-velocity accuracies; the numbers of stars observed to given accuracy levels; the diagnostic powers of the resulting data; and limitations arising from the complexities of the real sky.

\subsection{Astrometric accuracy}

There are three main components involved in the improved performance of GAIA compared with Hipparcos. The larger optics provide a smaller diffraction pattern and a significantly larger collecting area; the improved quantum efficiency and bandwidth of the detector (CCD rather than photocathode) leads to improved photon statistics; and use of CCDs provides an important multiplexing advantage.

The GAIA astrometric wavelength band $G$ is fixed such that $G \simeq V$ for un-reddened A0V stars. The approximate transformation from $(V, V-I)$ to $G$ can be expressed in the form:

$$
\begin{aligned}
G=V+0.51 & -0.50 \times \sqrt{0.6+(V-I-0.6)^{2}} \\
& -0.065 \times(V-I-0.6)^{2}
\end{aligned}
$$

which is valid (to $\pm 0.1 \mathrm{mag}$ ) at least for $-0.4<V-I \lesssim 6$. For $-0.4<V-I<1.4$ we have the convenient relation $G-V=0.0 \pm 0.1$ mag.

The sky background measured with HST at high ecliptic latitudes is $V=23.3 \mathrm{mag} \operatorname{arcsec}^{-1}$. Photometry and radial velocities calculations assume $V=$ $22.5 \mathrm{mag} \operatorname{arcsec}^{-2}$.

Many models of the Galaxy are available, from simplified star-count models to complex evolutionary models. For present purposes we adopt a star-count and kinematic model, updated from that of Chen (1997). This is probably satisfactory within $25 \%$, and is adequate until GAIA provides better data. The biggest uncertainty is perhaps the distribution of interstellar extinction. The model predicts that $\sim 1.1$ billion stars will be observable, corresponding to 1-2 per cent of the total stellar content of the Galaxy. The estimated number of stars according to the three Galactic populations (disk, thick disk and spheroid) shows that the main scientific goal, a representative census of the Galaxy, can be achieved. In addition, there are many rare objects of high astrophysical interest for which star count predictions cannot be obtained reliably from a Galaxy model. Details include globular clusters, double and multiple stars, high-density areas, and galaxies.

The basic accuracy estimation proceeds from the details of image formation, taking into account the detector signal, precision of the location estimator, instrument stability and calibration errors, and propagation from onedimensional (single-epoch) measurement errors to the final astrometric accuracy, estimated as:

$$
\sigma_{\mathrm{a}}=g_{\mathrm{a}}\left[\frac{\tau_{1}}{N_{i} \tau p_{\operatorname{det}}(G)}\left(\sigma_{\xi}^{2}+\sigma_{\text {cal }}^{2}\right)\right]^{1 / 2}
$$

where $N_{i}=2$ is the number of instruments, $\tau=L \Omega / 4 \pi$ the (average) total time available per object and instrument, $\tau_{1}$ is the integration time per CCD (s), $L$ is the effective mission length (s), $\sigma_{\xi}$ is the angular precision in the scan direction from one CCD crossing (rad), $\sigma_{\text {cal }}$ is the accuracy of the astrometric calibration, and $p_{\text {det }}(G)$ the detection probability as function of magnitude. The factor $g_{\mathrm{a}}$ relates the scanning geometry to the determination of the astrometric parameters. Numerical simulations of the scanning law are used to determine the mean values of $g_{\mathrm{a}}$ for given mission parameters, and their large-scale variations with ecliptic latitude. The estimation of $p_{\text {det }}$ is a complex problem, since it depends on many factors besides the brightness of the star. For the accuracy analysis an estimation of $p_{\text {det }}$ as function of $N$, the total number of photoelectrons in the stellar image, was made by means of dedicated simulations. An explicit error margin of 20 percent is added on the astrometric standard errors resulting from this analysis.

For the faintest stars the accuracy estimates take into account that a given star may not be observed in all transits. They include the factor $p_{\text {det }}(G)^{-1 / 2}$, where $p_{\text {det }}(G)$ is the detection probability as a function of the $G$ magnitude obtained from simulating star detection in the astrometric sky mapper using the robust APM algorithm. Since, by construction, the $G$ magnitude yields a rather uniform accuracy as a function of spectral type, useful mean accuracies can be derived from a straight mean of the detailed calculations, and are given in Table 8.

An approximate analytical fit to the tabular values for the parallax accuracy, $\sigma_{\pi}$, which also takes into account the slight colour dependence (due to the widening of the point spread function at longer wavelengths) is:

$\sigma_{\pi} \simeq\left(7+105 z+1.3 z^{2}+610^{-10} z^{6}\right)^{1 / 2} \times$

$$
[0.96+0.04(V-I)]
$$

where $z=10^{0.4(G-15)}$. For the position and proper motions errors, $\sigma_{0}$ and $\sigma_{\mu}$, the following mean relations can be used:

$$
\sigma_{0}=0.87 \sigma_{\pi}
$$$$
\sigma_{\mu}=0.75 \sigma_{\pi} \text {. }
$$

The expected standard errors vary somewhat over the sky as a result of the scanning law.

The number of stars whose distances can be determined to a certain relative accuracy can be estimated using a Galaxy model. For a given apparent magnitude and direction, the model provides the distribution of stars along the line of sight (in a small solid angle), as well as their distribution in colour index at each distance. It is 
Table 8. Mean accuracy in parallax $\left(\sigma_{\pi}\right)$, position (at mid-epoch, $\left.\sigma_{0}\right)$ and proper motion $\left(\sigma_{\mu}\right)$, versus $G$ magnitude. The values are sky averages

\begin{tabular}{lrrrrrrrrrrrr}
\hline$G(\mathrm{mag})$ & 10 & 11 & 12 & 13 & 14 & 15 & 16 & 17 & 18 & 19 & 20 & 21 \\
\hline$\sigma_{\pi}(\mu \mathrm{as})$ & 4 & 4 & 4 & 5 & 7 & 11 & 17 & 27 & 45 & 80 & 160 & 500 \\
$\sigma_{0}(\mu \mathrm{as})$ & 3 & 3 & 3 & 4 & 6 & 9 & 15 & 23 & 39 & 70 & 140 & 440 \\
$\sigma_{\mu}\left(\mu \mathrm{as} \mathrm{yr}{ }^{-1}\right)$ & 3 & 3 & 3 & 4 & 5 & 8 & 13 & 20 & 34 & 60 & 120 & 380 \\
\hline
\end{tabular}
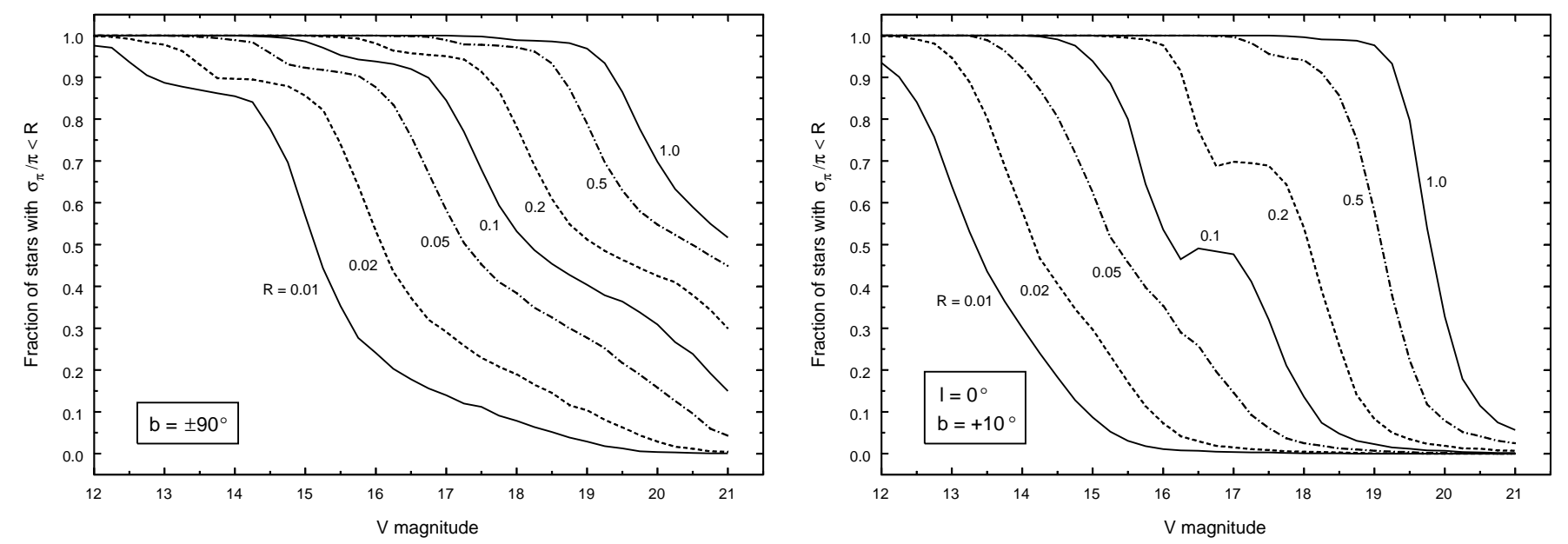

Fig. 6. Left: fraction of stars at a given magnitude having a relative parallax error less than 1, 2, 5, 10, 20, 50 and 100 percent, along the line of sight towards $\left(l=0^{\circ}, b=+90^{\circ}\right)$. Right: the same, for Galactic coordinates $\left(l=0^{\circ}, b=+10^{\circ}\right)$

then simple to compute the fraction of stars having relative parallax accuracy below a certain limit $R$. Figure 6 shows, for selected directions, the fraction of stars with relative parallax accuracies below $R=0.01-1.0$. Very good distance information $(R=0.1$ or better) will be obtained for virtually all stars brighter than $V=15$ and for significant fractions down to much fainter magnitudes, e.g. 10-50 percent at $V \sim 18$, depending on direction.

For $G \leq 15$ mag, more than 85 percent of the stars will have tangential velocity errors smaller than $5 \mathrm{~km} \mathrm{~s}^{-1}$, and 75 percent will be smaller than $2 \mathrm{~km} \mathrm{~s}^{-1}$. These figures worsen if all the stars with $G \leq 20 \mathrm{mag}$ are considered, but even in this case, for galactic latitudes higher than $5-10^{\circ}, 40$ percent of the stars observed by GAIA will have tangential velocities accurate to better than $10 \mathrm{~km} \mathrm{~s}^{-1}$.

\subsection{Photometric accuracy}

The photometric analysis cannot be separated from the astrometric analysis. The basic model for the pixel or sample values must be fitted simultaneously for the centroid coordinates $\xi_{0}, \eta_{0}$ and the photometric quantities $b$ and $N$. Moreover, this fitting must be done globally, by considering together all the transits of the object throughout the mission, in which the centroid positions are constrained by the appropriate astrometric model. This procedure is referred to as "global PSF fitting". A simpler procedure, "aperture photometry", is used for the photometric accuracy estimation.
Simple calculations for single-epoch accuracies including photon noise, read-noise and sky background noise have been adopted, and corrected with an error margin of 20 percent. The formal photometric precision reached at the end of the mission, typically by averaging $n_{\mathrm{obs}} \sim 50$ 100 observations, is on the level of one or a few millimag for the bright stars ( 12 mag). Can this be calibrated to reach this accuracy? Photometric calibration of the CCD zero points can be achieved from standards. The photometric consistency can be derived from repeated observations of all non-variable stars, and assured to a very high degree.

Photometric observations in a number of colour bands will be obtained for all stars detected. A quick photometric reduction will be carried out for each field crossing, providing rapid epoch photometry results for scientifically timecritical phenomena such as the detection of supernovae, burst, lensing, or other transient events. Improved photometric reduction can be obtained later in the mission, when accurate satellite attitude, CCD calibration data, and astrometric information for each star become available. This accuracy has been simulated using the complete GAIA image simulation and the APM photometry package. The two astrometric telescopes provide photometry in a wide spectral band defined by the CCD sensitivity. This $G$ band photometry from one single measure has a standard error of typically $0.01 \mathrm{mag}$ for $G \sim 18.5 \mathrm{mag}$. The two astrometric telescopes also provide broad-band photometry in four bands. The magnitudes resulting from averaging 67 observations obtained during a mission time 

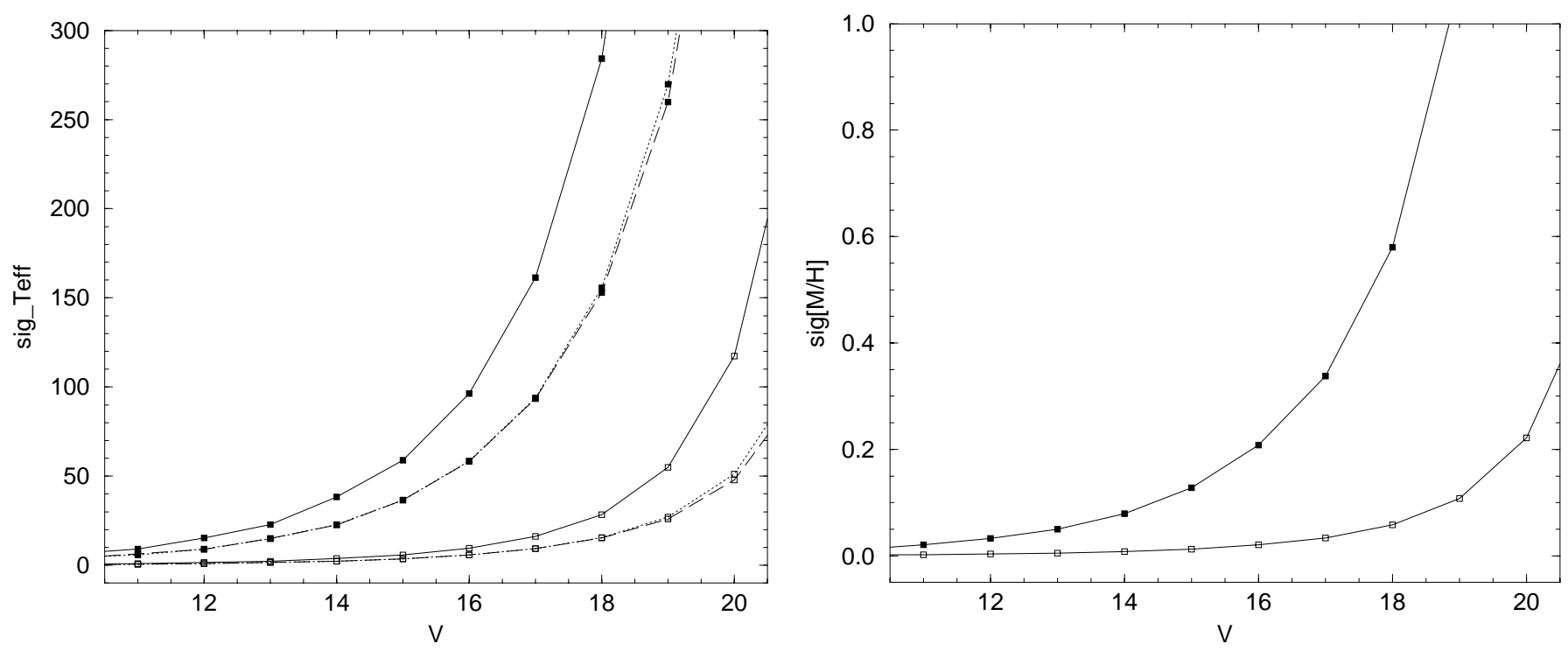

Fig. 7. Left: errors on $T_{\text {eff }}$ derived from C47-57 (continuous line), C57-75 (dotted line) and C75-89 (dashed line) indices, for M dwarfs with $T_{\text {eff }}=3500 \mathrm{~K}$. Solid symbols correspond to the errors for a single observation, open symbols to the mission-average (100 observations). Right: errors on $[\mathrm{Ti} / \mathrm{H}]$ derived from the $\mathrm{TiO}$ index, for $T_{\text {eff }}=3500 \mathrm{~K} \mathrm{M}$ dwarfs, for single observations (filled symbols) and for mission averages (open symbols)

of 4 years will have a precision of about $0.02 \mathrm{mag}$ in the F63B band (see Fig. 1) at $V \sim 20.0$ mag for all spectral types. The spectrometric telescope will collect photometry in 11 bands. The resulting average magnitudes will have a precision of $0.01 \mathrm{mag}$ in the $\mathrm{F} 57$ band at $V=19 \mathrm{mag}$, and $0.02 \mathrm{mag}$ in the F33 band for an unreddened G2V star.

The photometric accuracies as a function of colour index can be converted into accuracies on the astrophysical parameters. As an example, Fig. 7 (left) shows the uncertainty in effective temperature for G- and M-type dwarfs ( $T_{\text {eff }}=5750$ and $3500 \mathrm{~K}$ respectively) as a function of magnitude, for single transit and mission-average photometry, while Fig. 7 (right) shows the uncertainty in $[\mathrm{Ti} / \mathrm{H}]$ for M dwarfs.

\subsection{Radial velocity accuracy}

The radial velocity accuracy assessment has been derived from extensive numerical simulations, utilising observed spectra. Template spectra covering a wide range of astrophysical properties will be required. Global velocity zero points can be derived from the system geometry and astrometric positions of the target stars. The required accuracy is $1 / 40$ pixel for $1 \mathrm{~km} \mathrm{~s}^{-1}$. Performance has been studied using simulations as well as real observations of the selected spectral region for a variety of stars. Simulations were performed by producing synthetic spectra with different atmospheric parameters in the Ca II region, degrading them to the resolution and signal-to-noise ratio of a single GAIA observation, and then determining the radial velocity by cross-correlation. The photon budget was computed from the basic instrument design, and in addition to Poisson noise both the total read-out noise $\left(3 \mathrm{e}^{-}\right)$ and the sky background (normalized to a Solar spectrum with $V=22.5 \operatorname{arcsec}^{-2}$ ) were considered. For cool stars, the mission-average velocity accuracy is $\sigma_{v} \simeq 5 \mathrm{~km} \mathrm{~s}^{-1}$ at $V=18$, while for hot stars the performance is limited to $\sigma_{v} \simeq 10 \mathrm{~km} \mathrm{~s}^{-1}$ at $V=16 \mathrm{mag}$.

\subsection{Summary}

In summary, GAIA's measurement capabilities can be summarised as follows:

Catalogue: $\sim 1$ billion stars; $0.3410^{6}$ to $V=10 \mathrm{mag}$; $2610^{6}$ to $V=15 \mathrm{mag} ; 25010^{6}$ to $V=18 \mathrm{mag} ; 100010^{6}$ to $V=20 \mathrm{mag}$; completeness to about 20 mag.

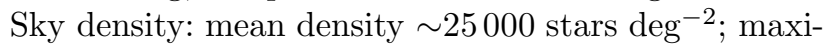
mum density $\sim 310^{6}$ stars $\mathrm{deg}^{-2}$.

Median parallax errors: $4 \mu$ as at 10 mag; $11 \mu$ as at $15 \mathrm{mag} ; 160 \mu$ as at $20 \mathrm{mag}$.

Distance accuracies: 2 million better than 1 percent; 50 million better than 2 percent; 110 million better than 5 percent; 220 million better than 10 percent.

Tangential velocity accuracies: 40 million better than $0.5 \mathrm{~km} \mathrm{~s}^{-1} ; 80$ million better than $1 \mathrm{~km} \mathrm{~s}^{-1} ; 200$ million better than $3 \mathrm{~km} \mathrm{~s}^{-1}$; 300 million better than $5 \mathrm{~km} \mathrm{~s}^{-1}$; 440 million better than $10 \mathrm{~km} \mathrm{~s}^{-1}$.

Radial velocity accuracies: $1-10 \mathrm{~km} \mathrm{~s}^{-1}$ to $V=$ 16-17 mag, depending on spectral type.

Photometry: to $V=20$ mag in 4 broad and 11 medium bands.

\section{Data analysis}

The total amount of (compressed) science data generated in the course of the five-year mission is about $210^{13}$ bytes (20 TB). Most of this consists of CCD raw or binned pixel values with associated identification tags. The data analysis aims to "explain" these values in terms of astronomical 


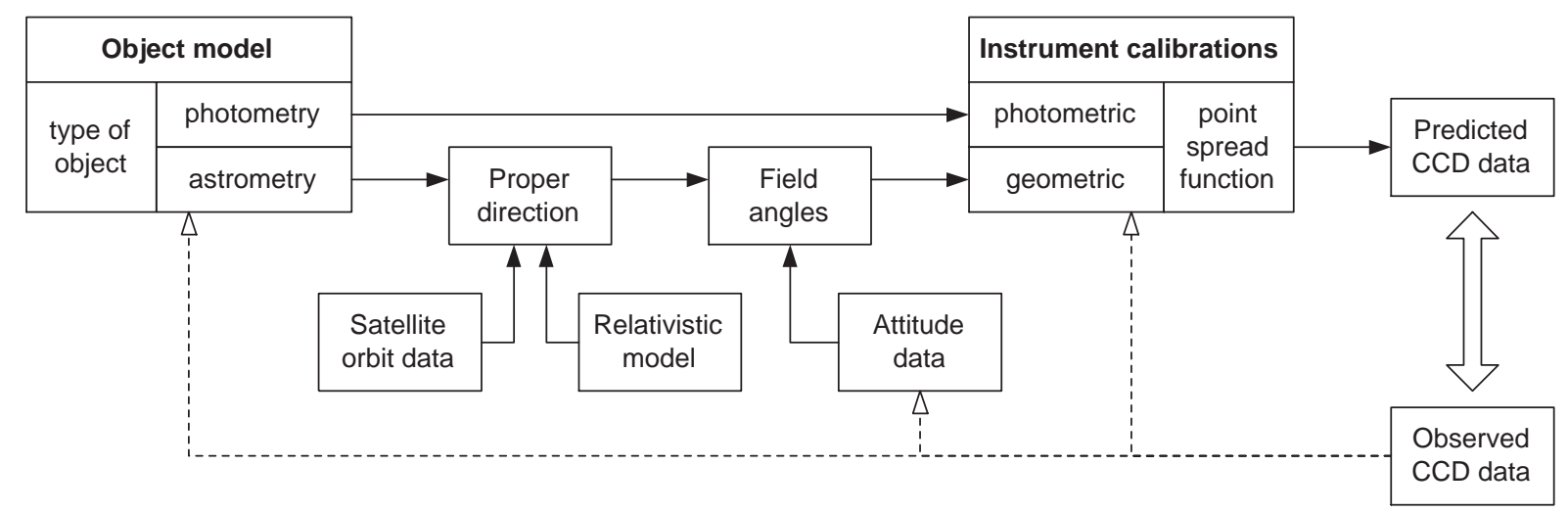

Fig. 8. Model of CCD data interrelations for an astronomical object. In principle, the data analysis aims to provide the "best" representation of the observed data in terms of the object model, satellite attitude and instrument calibration. Certain data and models can, from the viewpoint of the data analysis, be regarded as "given"; in the figure these are represented by the satellite orbit (in the barycentric reference system) and the relativistic model used to compute celestial directions. Other model data are adjusted to fit the observations (dashed lines)

objects and their characteristics. In principle the analysis is done by adjusting the object, attitude and instrument models until a satisfactory agreement is found between predicted and observed data (dashed lines in Fig. 8). Successful implementation of the data analysis task will require expert knowledge from several different fields of astronomy, mathematics and computer science to be merged in a single, highly efficient system (O'Mullane \& Lindegren 1999).

The global astrometric reductions must be formulated in a fully general relativistic framework, including postpost-Newtonian effects of the spherical Sun at the $1 \mu$ as level, as well as including corrections due to oblateness and angular momentum of Solar System bodies.

Processing these vast amounts of data will require highly automated and efficient numerical methods. This is particularly critical for the image centroiding of the elementary astrometric and photometric observation in the astrometric instruments, and the corresponding analysis of spectral data in the spectrometric instrument.

Accurate and efficient estimation of the centroid coordinate based on the noisy CCD samples is crucial for the astrometric performance. Simulations indicate that 6 samples approximately centred on the peak can be read out from the CCD. The centroiding, as well as the magnitude estimation, must be based on these six values. Results of a large number of Monte Carlo experiments, using a maximum-likelihood estimator as the centroiding algorithm, indicate that a rather simple maximum-likelihood algorithm performs extremely well under these idealized conditions, and that six samples is sufficient to determine the centroid accurately. Much work remains to extend the analysis to more complex cases, including in particular overlapping stellar images.

A preliminary photometric analysis, for discovery of variables, supernovae, etc., can be carried out using standard photometric techniques immediately after data delivery to the ground. In addition, more detailed modelling of the local background and structure in the vicinity of each target using all the mission data in all the passbands will be required. A final end-of-mission re-analysis may benefit from the astrometric determination of the image centroids, locating a well-calibrated point spread function for photometric analysis. Studies of these photometric reductions have begun.

The high-resolution (radial velocity) spectrometer will produce spectra for about a hundred million stars, and multi-epoch, multi-band photometry will be obtained for about one billion stars. The analysis of such large numbers of spectra and photometric measurements needs to be performed in a fully automated fashion, with no manual intervention. Automatic determination of (at least) the surface temperature $T_{\text {eff }}$, the metallicity $[\mathrm{M} / \mathrm{H}]$, and the relative $\alpha$ element abundance $[\alpha / \mathrm{Fe}]$ is necessary; determination of $\log g$ is, given the availability of parallaxes for most stars, of lesser importance. A fully automated system for the derivation of astrophysical parameters from the large number of spectra and magnitudes collected by GAIA, using all the available information for each star, has been studied, showing the feasibility of an approach based on the use of neural networks. In the classification system foreseen, spectra and photometric measurements will be sent to an "initial classifier", to sort objects into stellar and non-stellar. Specialist networks then treat each class. For example, stellar data sets are passed to an "automated stellar parameterization" sub-package.

It is the physical parameters of stars which are really of interest; therefore the proposed system aims to derive physical parameters directly from a stellar spectrum and photometry. Detailed simulations of the automated stellar parameterization system have been completed using a feed-forward neural network operating on the entire set of spectral and photometric measurements. In such a system, the derived values for the stellar parameters are naturally linked to the models used to train the network. Given the extreme rapidity of neural networks, when stellar atmosphere models are improved, re-classification of the entire data set can be done extremely quickly: an archive of 
$10^{8}$ spectra or photometric measurements could be reclassified in about a day with the present-day computing power of a scientific workstation.

The overall data analysis task would be impossible without certain regularizing assumptions: one must assume that a substantial fraction of stars follow a very simple model, viz. (apparently) single stars with little or no photometric variability, whose motions can be described by the standard five astrometric parameters $\left(\alpha, \delta, \pi, \mu_{\alpha *}\right.$, $\left.\mu_{\delta}\right)$. For the satellite attitude and instrument characteristics it must be assumed that sudden changes are rare, so that time-averaging and smoothing are effective in reducing observational noise. Without these assumptions the problem would simply have too many degrees of freedom. While such regularity conditions must be valid in a broad sense, it is clear that they cannot be guaranteed to hold in any particular situation or for a specific object. The data analysis must be able to filter out cases where the conditions do not apply, and divert them to a separate analysis branch. The efficiency of the filtering process depends critically on the quality of the instrument calibrations and attitude determination, which initially is quite low. Thus an iterative process is needed in which the object selection and observations are successively improved, along with the calibrations and attitude determination.

The computational complexity of the data analysis arises not just from the amount of data to be processed, but even more from the intricate relationships between the different pieces of information gathered by the various instruments throughout the mission. It is difficult to assess the magnitude of the data analysis problem in terms of processing requirements. Certain basic algorithms that have to be applied to large data sets can be translated into a minimum required number of floating-point operations. Various estimates suggest of order $10^{19}$ floating-point operations, indicating that very serious attention must be given to the implementation of the data analysis, and that this effort must start very early.

Observations of each object are distributed throughout the mission, so that calibrations and analysis must be feasible both in the time-domain and in the object domain. Flexibility and interaction is needed to cope with special objects, while calibrations must be protected from unintentional modification. Object Oriented (OO) methodologies for data modeling, storage and processing are ideal for meeting the challenges faced by GAIA.

The feasibility of the OO approach has been demonstrated by a short prototyping exercise carried out during the present study phase. Algorithms for three processes were provided and incorporated into the OO model, underlining one important feature of OO design: the ability to have complex data structures and operations described in a single model. Java code was generated from the model and the algorithms implemented. The prototype was highly successful and reinforced confidence in the OO approach for treating the data. The reduction process is inherently distributed, and naturally matched to distributed parallel processors.
Table 9. Summary of the capabilities of Hipparcos and GAIA, along with those of the DIVA (Germany) and SIM and FAME (NASA) astrometric space missions. Numbers of stars are indicative; in the case of SIM they are distributed amongst grid stars and more general scientific targets. Typical accuracies are given according to magnitude where appropriate

\begin{tabular}{lcrclc}
\hline Mission & Launch & $\begin{array}{r}\text { No. of } \\
\text { stars }\end{array}$ & $\begin{array}{c}\text { Mag } \\
\text { limit }\end{array}$ & \multicolumn{2}{c}{ Accuracy } \\
& & 120000 & 12 & 1 & 10 \\
& Hipparcos & 1989 & mag) \\
DIVA & 2004 & 40 million & 15 & 0.2 & 9 \\
& & & & 5 & 15 \\
FAME & 2004 & 40 million & 15 & 0.050 & 9 \\
& & & & 0.300 & 15 \\
SIM & 2009 & 10000 & 20 & 0.003 & 20 \\
GAIA & 2012 & 1 billion & 20 & 0.003 & 12 \\
& & & & 0.010 & 15 \\
& & & & 0.200 & 20 \\
\hline
\end{tabular}

\section{GAIA and other space missions}

The scientific capabilities and goals of GAIA and other proposed or approved space astrometric missions are summarised in Table 9. GAIA is a survey mission, essential for statistical analysis of the unknown, with broad applications to the Solar System, galaxies, large-scale structure, and primarily Galactic structure and evolution. SIM (Space Interferometry Mission, Boden et al. 1997) is an interferometer, ideal for precise measurements of a small number of carefully pre-selected targets of specific scientific interest. The SIM target selection is yet to happen, but will be focussed towards searches for low-mass planets around a few nearby stars, calibration of the distance scale, and detailed studies of known micro-lensing events.

FAME (Full-Sky Astrometric Mapping Explorer; Triebes et al. 2000), selected by NASA in the MIDEX competition in 1999, and DIVA (Double Interferometer for Visual Astrometry; Röser et al. 1997), a small German astronomy satellite planned for launch in 2004, are essentially successors to Hipparcos, with an extension of limiting sensitivity, sample size and accuracy (statistical weight) by a factor of order 100 in each. DIVA and FAME will both provide an excellent reference frame, substantially improve calibration of the distance scale and the main phases of stellar evolutionary astrophysics, and map the Solar Neighbourhood to much improved precision. The difference between GAIA and these two missions is one of scale and comprehensiveness. GAIA exceeds these two missions in scale by a further factor of order 100, allowing study of the entire Galaxy, and only GAIA will provide photometric and radial velocity measurements as crucial astrophysical diagnostics.

\section{Conclusion}

GAIA will create an extraordinarily precise threedimensional map of about one billion stars throughout our 
Galaxy and the Local group. It will map their space motions, which encode the origin and subsequent evolution of the Galaxy, and the distribution of dark matter. Through on-board photometry, it will provide the detailed physical properties of each star observed: luminosity, temperature, gravity, and elemental composition, which encode the star formation and chemical enrichment history of the Galaxy. Radial velocity measurements on board will complete the kinematic information for a significant fraction of the objects observed.

Through continuous sky scanning, the satellite will repeatedly measure positions and colours of all objects down to $V=20 \mathrm{mag}$. On-board object detection ensures a complete census, including variable stars and quasars, supernovae, and minor planets. It also circumvents costly pre-launch target selection activities. Final accuracies of 10 microarcsec at $15 \mathrm{mag}$ will provide distances accurate to 10 percent as far as the Galactic Centre. Stellar motions will be measured even in the Andromeda galaxy.

In order to limit failure modes, the instrument design includes only one deployable element (the sun shield/solar array) and only three on-board mechanisms (two secondary mirror correctors, one for each of the astrometric telescopes, and an orientation adjustment for the spectroscopic CCDs). Operation of the focal plane is robust against failure of individual CCDs, or against failure of one or more "rows" of the main focal plane (whether it be the sky mapper, the main detectors, or the on-board data handling units associated with each such row), which would correspond to no more than a loss of overall observing time. The CCDs for the medium-band photometer and radial velocity spectrograph are designed so that the loss if one CCD leads to the loss of only the upper or lower half of one colour band, amounting to a "graceful degradation" of the mission's science goals. Loss of throughput, of whatever form, would lead to a decrease in the limiting magnitude, and a corresponding degradation of the astrometric accuracy as a function of magnitude. It is not easy to quantify "break points" in the scientific case at which the astrometric improvement compared to Hipparcos, occasioned by failures or performance limitations, would cease to have a significant scientific impact, as evidenced by the selection of both the FAME and DIVA missions. Nonetheless, a limiting magnitude of $20 \mathrm{mag}$, accuracies of $10 \mu \mathrm{arcsec}$ at $15 \mathrm{mag}$, and the provision of in-depth photometric and radial velocity data for each object, remain primary mission goals.

GAIA's main scientific objective is to clarify the origin and history of our Galaxy, from a quantitative census of the stellar populations. It will advance fundamental questions such as when the stars in the Milky Way formed, when and how the Milky Way was assembled, and the distribution of dark matter in our Galaxy. In so doing, it will pinpoint exotic objects in substantial numbers: many thousands of extra-Solar planets will be discovered, and their detailed orbits determined; tens of thousands of brown dwarfs and white dwarfs will be identified; rare stages of stellar evolution will be quantified; some
100000 extragalactic supernovae will be discovered, and details communicated for follow-up ground-based observations; Solar System studies will receive a massive impetus through the detection of many tens of thousands of new minor planets; inner Trojans and even new transNeptunian objects, including Plutinos, may be discovered. GAIA will follow the bending of star light by the Sun and major planets over the entire celestial sphere, and therefore directly observe the structure of space-time - the accuracy of its measurement of general relativistic light bending may reveal the long-sought scalar correction to its tensor form. The PPN parameters $\gamma$ and $\beta$, and the Solar quadrupole moment $J_{2}$, will be determined with unprecedented precision. New constraints on the rate of change of the gravitational constant, $\dot{G}$, and on gravitational wave energy over a certain frequency range, will be obtained.

GAIA is timely as it complements other major space and ground initiatives. Understanding and exploration of the early Universe, through microwave background studies (Planck) and direct observations of high-redshift galaxies (NGST, FIRST, ALMA), are complemented by theoretical advances in understanding the growth of structure from the early Universe up to galaxy formation. Serious further advances require a detailed understanding of a "typical" galaxy, to test the physics and assumptions in the models. Our Galaxy, a typical example of those luminous spirals which dominate the luminosity of the Universe, uniquely provides such a template.

Acknowledgements. This summary of the GAIA mission, as presented to and approved by the scientific advisory committees of the European Space Agency in September-October 2000 , is based on the GAIA Study Report, which is the result of a large collaboration between ESA, the European scientic community and European industry. The scientific aspects of the study were supervised by the Science Advisory Group Members, comprised by the authors. The work of the Science Advisory Group has been supported by a Science Working Group, chaired by P. T. de Zeeuw and G. Gilmore (18 members) and responsible for quantifying the science case; a Photometry Working Group, chaired by F. Favata (18 members); an Instrument Working Group, chaired by L. Lindegren (16 members); and 52 other European scientists directly supporting the GAIA study. We gratefully acknowledge the many and detailed contributions made by the working group members, as well as guidance on the content of this paper from the referee, Donald J. Hutter.

Technological activities have been led by the ESA Study Manager, O. Pace, supported by M. Hechler (ESOC Study Manager), and ESA-ESTEC engineers. ESA scientific involvement includes contributions from S. Volonté (ESA Paris) and from scientists within the ESA Astrophysics Division (K. S. O'Flaherty, F. Favata, W. O'Mullane, M. Vannier, and A. Colorado McEvoy).

The satellite design study has been under contract to Astrium (formerly Matra Marconi Space, F), under Study Manager P. Mérat, and involving EEV Ltd (UK), and Alcatel Space (F). An Alenia Study Team, under Study Manager S. Cesare, evaluated the performance of an interferometric design, with involvement of the Istituto di Metrologia "G. Colonnetti", EICAS Automazione, the Osservatorio 
Astronomico di Torino, Matra Marconi Space (F), and Alcatel Space. Other industrial studies have been carried out by SIRA (UK; CCD CTE), and TNO-TPD (Delft; basic angle monitoring).

\section{References}

Battrick, B. 1994, in Horizon 2000 Plus: European Space Science in the 21st Century, ed. B. Battrick, ESA SP-1180, ESA, Noordwijk

Boden, A., Unwin, S., \& Shao, M. 1997, in Hipparcos Venice 97, ed. M. A. C. Perryman, \& P. L. Bernacca, ESA SP-402, ESA, Noordwijk, 789

Chen, B. 1997, ApJ, 491, 181

de Zeeuw, P. T. 1999, in The Third Stromlo Symposium: The Galactic Halo: Bright Stars and Dark Matter, ed. B. K. Gibson, T. S. Axelrod, \& M. E. Putman, ASP Conf. Ser. 165,515

ESA 2000, GAIA: Composition, Formation and Evolution of the Galaxy, Technical Report ESA-SCI(2000)4, (scientific case on-line at http://astro.estec.esa.nl/GAIA)

Eyer, L., \& Cuypers, J. 2000, in The Impact of Large-Scale Surveys on Pulsating Star Research, ed. L. Szabados, \& D. W. Kurtz, ASP Conf. Ser. 203, Astronomical Society of the Pacific, San Francisco, 71

Feissel, M., \& Mignard, F. 1998, A\&A, 331, L33

Freeman, K. C. 1993, in Galaxy Evolution: The Milky Way Perspective, ed. S. Majewksi, ASP Conf. Ser. 49, 125

Gilmore, G. 1999, Baltic Astron. 8, 203

Gilmore, G., de Boer, K. S., Favata, F., et al. 2000, in UV, Optical and IR Space Telescopes and Instruments, ed. J. B. Brekinridge, \& P. Jakobsen, SPIE 4013

Gilmore, G., \& Høg, E. 1995, in Future Possibilities for Astrometry in Space, ed. M. A. C. Perryman, \& F. van Leeuwen, ESA SP-379, ESA, Noordwijk, 95

Gilmore, G., Wyse, R. F. G., \& Kuijken, K. 1989, ARA\&A, 27,555

Hernandez, X., Gilmore, G., \& Valls-Gabaud, D. 2000, MNRAS, 316, 605

Høg, E. 1993, in Developments in Astrometry and their Impact on Astrophysics and Geodynamics, IAU Symp. 156, ed. I. I. Mueller, \& B. Kolaczek (Kluwer, The Netherlands), 37

$\mathrm{H} \varnothing \mathrm{g}$, E. 1995a, in Astronomical and Astrophysical Objectives of Sub-Milliarcsec Astrometry, IAU Symp. 166, ed. E. Høg, \& P. K. Seidelmann (Kluwer), 317

Høg, E. 1995b, in Future Possibilities for Astrometry in Space, ed. M. A. C. Perryman, \& F. van Leeuwen, ESA SP-379, ESA, Noordwijk, 263

Høg, E., Fabricius, C., Knude, J., \& Makarov, V. V. 1999a, Baltic Astron., 8, 25

Høg, E., Fabricius, C., \& Makarov, V. V. 1999b, Baltic Astron.,
8,233

Høg, E., \& Lindegren, L. 1993, in Developments in Astrometry and their Impact on Astrophysics and Geodynamics, IAU Symp. 156, ed. I. I. Mueller, \& B. Kolaczek (Kluwer, The Netherlands), 31

Høg, E., \& Lindegren, L. 1994, in Galactic and Solar System Optical Astrometry, ed. L. V. Morrison, \& G. Gilmore (Cambridge University Press), 246

Ibata, R. A., Wyse, R. F. G., Gilmore, G., Irwin, M., \& Suntzeff, N. 1997, AJ, 113, 634

Irwin, M. J. 1985, MNRAS, 214, 575

Johnston, K. J., \& de Vegt, C. 1999, ARA\&A, 37, 97

Lattanzi, M. G., Spagna, A., Sozzetti, A., \& Casertano, S. 2000, MNRAS, 317, 211

Lebreton, Y. 2000, ARA\&A, 38, 35

Lindegren, L., Bastian, U., Gilmore, G., et al. 1993a, Roemer: Proposal for the Third Medium Size ESA Mission (M3), Technical Report, Lund Observatory

Lindegren, L., \& Perryman, M. A. C. 1996, A\&AS, 116, 579

Lindegren, L., Perryman, M. A. C., Bastian, U., et al. 1993b, GAIA - Proposal for a Cornerstone Mission concept submitted to ESA in October 1993, Technical Report, Lund

Majewski, S. 1993, ARA\&A, 31, 575

Marcy, G. W., \& Butler, R. P. 1998, in Cool Stars, Stellar Systems and the Sun; Proceedings of the 10th Cambridge Workshop, ed. R. A. Donahue, \& J. A. Bookbinder, ASP Conf. Ser. 154, San Francisco, 9

Mérat, P., Safa, F., Camus, J. P., Pace, O., \& Perryman, M. A. C. 1999, Baltic Astron., 8, 1

Mignard, F. 1999, in Harmonizing Cosmic Distance Scales in a Post-Hipparcos Era, ed. D. Egret, \& A. Heck, ASP Conf. Ser. 167,44

Munari, U. 1999a, Baltic Astron., 8, 73

Munari, U. 1999b, Baltic Astron., 8, 123

O'Mullane, W., \& Lindegren, L. 1999, Baltic Astron., 8, 57

Paczyński, B. 1997, in Proc. 12th IAP Astrophysics Coll. 1996: Variable Stars and Astrophysical Returns from Microlensing Surveys, ed. R. Ferlet, J. P. Maillard, \& B. Raban (Éditions Frontières), 357

Perryman, M. A. C. 2000, Rep. Prog. Phys., 63, 1209

Röser, S., Bastian, U., de Boer, K. S., et al. 1997, in Hipparcos Venice 97, ESA SP-402, ESA, ed. M. A. C. Perryman, \& P. L. Bernacca (Noordwijk), 777

Söderhjelm, S. 1999, A\&A, 341, 121

Straizys, V. 1999, Baltic Astron., 8, 109

Triebes, K. J., Gilliam, L., Hilby, T., et al. 2000, SPIE 4013, 482

Vaccari, M. 2000, GAIA Galaxy Survey, Master Thesis, University of Padova

Wyse, R. F. G., Gilmore, G., \& Franx, M. 1997, ARA\&A, 35, 637 